\title{
Origin of texture development in orthorhombic uranium
}

\author{
Miroslav Zecevic ${ }^{\mathrm{a}}$, Marko Knezevic ${ }^{\mathrm{a}, *}$, Irene J. Beyerlein ${ }^{\mathrm{b}}$, Rodney J. McCabe \\ ${ }^{a}$ Department of Mechanical Engineering, University of New Hampshire, Durham, NH 03824, USA \\ ${ }^{\mathrm{b}}$ Theoretical Division, Los Alamos National Laboratory, Los Alamos, NM 87545, USA \\ ${ }^{\mathrm{c}}$ Materials Science and Technology Division, Los Alamos National Laboratory, Los Alamos, NM 87545, USA
}

\begin{abstract}
We study texture evolution of alpha-uranium $(\alpha-\mathrm{U})$ during plane strain compression and uniaxial compression to high strains at different temperatures. We combine a multiscale polycrystal constitutive model and detailed analysis of texture data to uncover the slip and twinning modes responsible for the formation of individual texture components. The analysis indicates that during plane strain compression, floor slip (001)[100] results in the formation of two pronounced $\{001\}$ texture peaks tilted $10-15^{\circ}$ away from the normal toward the rolling direction. During both high-temperature (573K) through-thickness compression and plane strain compression, the active slip modes are floor slip (001)[100] and chimney slip $1 / 2\{110\}\langle 1 \overline{1} 0\rangle$ with slightly different ratios. $\{130\}\langle 3 \overline{1} 0\rangle$ deformation twinning is profuse during rolling and in-plane compression and decreases with increasing temperature, but is not as active for throughthickness compression. Finally, we comment on some similarities between rolling textures of $\alpha$ $\mathrm{U}$, which has a c/a ratio of 1.734 , and those that develop in hexagonal close packed metals with similarly high c/a ratios like $\mathrm{Zn}$ (1.856) and $\mathrm{Cd}$ (1.885) and are dominated by basal slip.
\end{abstract}

Keywords: Orthorhombic structure; Uranium; Texture; Rolling; Crystal plasticity

${ }^{*}$ Corresponding author at: Department of Mechanical Engineering, University of New Hampshire, 33 Academic Way, Kingsbury Hall, W119, Durham, New Hampshire 03824, USA. Tel.: +1 603862 5179; fax: +1 603862 1865; E-mail address: marko.knezevic@unh.edu 


\section{Introduction}

The plastic deformation of polycrystalline alpha-uranium $(\alpha-U)$ is governed by slip and twinning. These mechanisms occur on different crystallographic planes and directions $[1,2]$. Not only does each mode have its own activation stresses but also separate dependencies on temperature and strain rate $[3,4]$. How the grain microstructure of $\alpha$-U, including grain sizes and shapes, crystal orientations (texture), and defect densities, evolves in deformation is governed by which and what proportion of slip and twinning modes are activated. The mechanical performance of $\alpha-\mathrm{U}$, its strength, flow stress evolution with strain, and plastic anisotropy, are highly sensitive to the details of its texture. For this reason, it is desirable to understand texture evolution during bulk forming processing of $\alpha-U$ with the aim towards controlling its mechanical behavior.

Experimental and modeling studies have shown that the development of texture in $\alpha-\mathrm{U}$ highly depends on the initial texture, deformation path, strain rate, and temperature [5-8]. Via polycrystal modeling, it has been demonstrated that predicting texture evolution relies on the ability to model which slip and twinning modes are active at any given point in the deformation process [9]. These studies account for the cooperation of many slip and twinning systems acting simultaneously over the history of deformation. However, since so many deformation modes are active at once, it is difficult to discern which ones are responsible for particular components in the bulk texture.

For low symmetry crystal structures, such as hexagonal close packed, the effects of a certain slip mode on a particular aspect of texture evolution has been studied [10-19]. However, similar analyses on metals with an orthorhombic crystal structure have not been conducted. In this work, we employ a set of polycrystal plasticity models to understand the relationship between the development of common texture components and the main underlying deformation mechanisms in $\alpha-U$ during both uniaxial compression and large rolling reductions. For this analysis, the models use the same set of material-dependent parameters to predict the mechanical response and texture evolution in simple compression and rolling to large strains from room temperature to $573 \mathrm{~K}$. We discuss the origin of texture evolution in rolling and compare it with texture evolution during simple compression. 


\section{Material, processing, and experimental methods}

The $\alpha-\mathrm{U}$ material used in this study was initially cast into ingots using vacuum-inductionmelting [5, 20]. These ingots were first upset forged and hot rolled at $640 \mathrm{C}$ and then annealed for two hours at $480 \mathrm{C}$. They were warm straight-rolled at $330 \mathrm{C}$ to a final sheet thickness of $8.89 \mathrm{~mm}$. Last, the material was annealed at $550 \mathrm{C}$ for two hours. The final microstructure after these pre-processing steps constitutes the initial microstructure for our deformation studies carried out here.

Electron backscattered diffraction (EBSD) was used to characterize the texture [5, 20]. Specimens were metallographically prepared through a sequence of mechanical grinding and polishing followed by electropolishing [20]. EBSD data was collected using a TSL/EDAX EBSD camera attached to a FEI XL30 ESEM at an accelerating voltage of 25KV. EBSD scans with 5-10 $\mu \mathrm{m}$ step sizes over an area of several square millimeters were used for texture measurements. The objective of these texture scans was to sample a large number of grains, and not necessarily to generate microstructural statistics or even high confidence index data. Nevertheless, the average confidence index of the raw data scans used to generate the initial texture was 0.59 , and data points with the confidence index (CI) [21] below 0.5 were discarded prior to calculating the texture. The initial texture of the material is shown in Fig. 1a. The initial grain size was around $15 \mu \mathrm{m}$ and the initial texture is based on sampling of around 30,000 different grains. This starting texture exhibits a moderate (001) component in the throughthickness direction (TT), (010) component in the in-plane 1-direction (IP1) and (100) component in the other in-plane direction (IP2). Figure $1 \mathrm{~b}$ shows a random texture also used in analysis presented later in the paper.

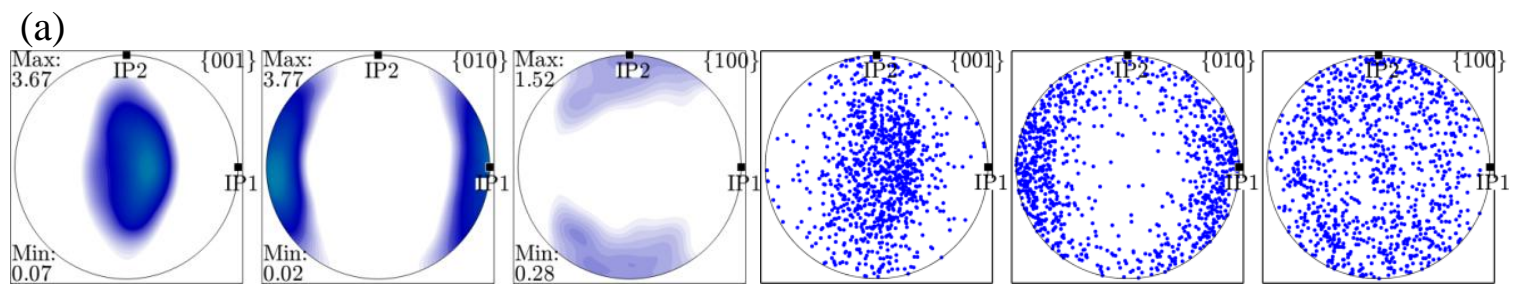

(b)

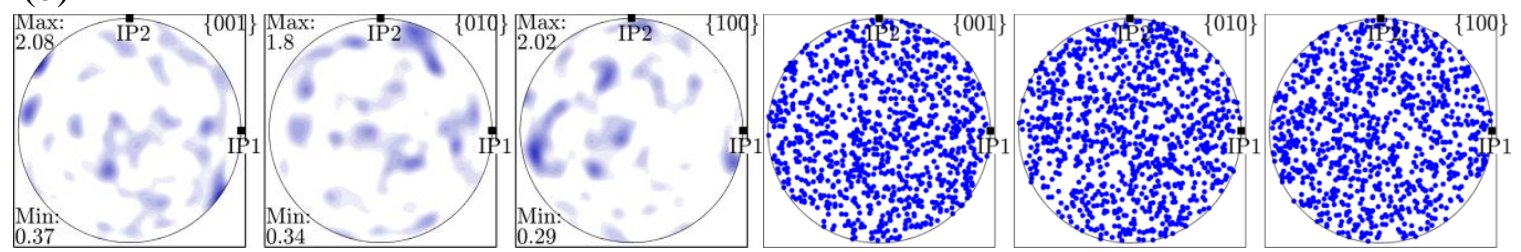




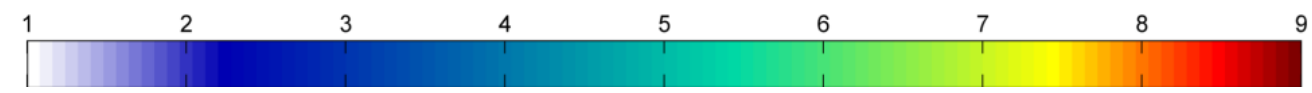

Fig. 1. (a) Pole figures showing the initial textures measured in the as-annealed samples of straight rolled uranium. IP1 is the original straight-rolling direction of the plate. The multiple random intensity plot (on the left) and point plot (on the right) pole figures are plotted using 10,000 and 1,000 crystal orientations, respectively. (b) Pole figures for an initial random texture is represented using 1000 discrete orientations.

The material was tested in uniaxial compression at different temperatures ranging from room temperature to $573 \mathrm{~K}$ [5] or further rolled to large strains at $573 \mathrm{~K}$. In the latter case, rolling took place $90^{\circ}$ to the original straight rolling direction, i.e. in the IP2 direction, in several rolling passes reducing the plate thickness from $8.89 \mathrm{~mm}$ to $3.56 \mathrm{~mm}$. EBSD was used to measure the texture following $16 \%$ rolling reduction to a thickness of $5.96 \mathrm{~mm}, 44 \%$ rolling reduction to a thickness of $4.27 \mathrm{~mm}$, and $60 \%$ rolling reduction to a final thickness of $3.56 \mathrm{~mm}$,.

\section{Multiscale modeling approach}

To understand, analyze, and interpret texture evolution of alpha-uranium, we employ a multiscale crystal plasticity based model for the constitutive behavior of a polycrystalline material that deforms by slip and twinning. The model was developed in [22] and in that work, was applied to $\mathrm{Zr}$, which like uranium deforms by multiple slip and twinning modes. Below we provide an abbreviated description of the model.

At the largest scale, the model uses the visco-plastic self-consistent (VPSC) polycrystal plasticity model [23]. In VPSC, a polycrystal is represented by a number of grains, each with an assigned crystallographic orientation, shape and volume fraction. The grain stress and strain are calculated by treating each grain as a visco-plastic inclusion embedded in a homogeneous effective medium (HEM) [23-26]. The HEM has anisotropic plastic properties equal to the averaged anisotropic plastic properties of all grains.

To model the effect of the constraint of neighboring grains on grain reorientation, we invoked the co-rotation scheme [27]. In this scheme, each grain is assigned one or more randomly chosen grain(s) in the polycrystal to serve as its neighbor(s). Then the average reorientation of grain and its neighbor(s), weighted by their volume fractions, is calculated and used for grain rotation [28]. 
Each inclusion (or representative grain) deforms only by plasticity via a combination of crystallographic slip and twinning systems. The Cauchy stress deviator $\boldsymbol{\sigma}^{\prime}$ and the viscoplastic strain-rate $\dot{\boldsymbol{\varepsilon}}_{v p}$ of a grain are related by:

$$
\dot{\boldsymbol{\varepsilon}}_{v p}(\mathbf{x})=\sum_{s=1}^{N_{S}} \mathbf{m}^{s}(\mathbf{x}) \dot{\gamma}^{s}(\mathbf{x}),
$$

where $N_{s}$ is the total number of slip and twin systems. The slip or twin shearing rate, $\dot{\gamma}^{s}$, on a system, $s$, is related to the resolved shear stress, $\tau^{s}$, on that system by [29-31]:

$$
\dot{\gamma}^{s}=\dot{\gamma}_{0}\left|\frac{\boldsymbol{\sigma}^{\prime} \cdot \mathbf{m}^{s}}{\tau_{c}^{s}}\right|^{\frac{1}{m}} \operatorname{sign}\left(\boldsymbol{\sigma}^{\prime} \cdot \mathbf{m}^{s}\right),
$$

where $\dot{\gamma}_{0}$ is the reference slip rate set to the norm of the imposed strain rate, $m$ is the rate sensitivity parameter, $\mathbf{m}^{s}$ is the Schmid tensor for slip or twinning system $s$ and $\tau_{c}^{s}$ is the threshold stress on the system. Here we set $\dot{\gamma}_{0}$ to be the macroscopic strain rate rendering the model to be rate independent [32] and $1 / m$ to be 10 .

To model lattice reorientation due to deformation twinning at the grain scale, we employ the composite grain model (TW) $[13,33]$. The formation of a twin domain within a grain with a characteristic twin-matrix orientation relationship is modeled by partitioning the grain into a representative twin and matrix inclusion [33]. A newly formed twin inclusion is assigned an initial morpholopy and lattice orientation. The crystallographic orientation of the twin domain corresponds to the predominant twin system (PTS), which is the twinning system with the highest shear-rate among all active twin systems. The short axes of the matrix ellipsoids are perpendicular to the twin plane. The long axes of both are assigned the grain size $d_{g}$ and the short axis of the matrix is assigned $d_{c}=\frac{d_{g}}{n^{\text {lamellae }}}$, which is the separation between adjacent twin lamellae. The number $n^{\text {lamellae }}$ is the number of twin lamellae per grain. In the present work, it is set equal to two, an average value seen experimentally. To model twin growth, the ellipsoids representing the twins are allowed to thicken. At the same time, those representing the matrix shrink.

In this model, the threshold stresses for slip and twinning evolve with strain and their evolutions follow different rate laws. For slip, the threshold stresses are a sum of many contributions [11, 34-36]:

$$
\tau_{c}^{s}=\tau_{0}^{\alpha}+\tau_{\text {for }}^{s}+\tau_{\text {deb }}^{\alpha}
$$


The first $\tau_{0}^{\alpha}$ is an initial slip resistance. An empirical model is used for $\tau_{0}^{\alpha}(T)$, which varies with temperature via:

$$
\tau_{0}^{\alpha}(T)=A \exp \left(-\frac{T-295}{B}\right),
$$

where $T$ is temperature in Kelvin, $A$ is the room temperature $(295 \mathrm{~K})$ resistance, and $B$ is a material constant. The barrier effect of the initial grain size was not characterized separately and thus is simply included in the initial slip resistance. This is possible since $\tau_{0}^{\alpha}$ includes all contributions reflecting the initial microstructure such as Peierls stress, an initial content of dislocations as well as the grain size barrier term.

The second and third terms $\tau_{\text {for }}^{S}$ and $\tau_{d e b}^{\alpha}$ in Eq. (3) are governed by the evolution of stored dislocation density on the slip system $s$ according to thermally activated rate laws and hence are explicit functions of temperature and strain rate [4, 22, 35, 37-39]. First, the resistances arising from stored dislocations, $\tau_{\text {for }}^{S}(\dot{\varepsilon}, T)$ and $\tau_{\text {deb }}^{\alpha}(\dot{\varepsilon}, T)$, are directly related to the stored forest $\rho_{f o r}^{S}(\dot{\varepsilon}, T)$ and debris $\rho_{d e b}(\dot{\varepsilon}, T)$ dislocation densities via Taylor-type laws $[40,41]$ according to:

$$
\begin{aligned}
& \tau_{f o r}^{S}(\dot{\varepsilon}, T)=b^{\alpha} \mu^{\alpha}(T) \sqrt{\chi^{S S \prime} \rho_{f o r}^{S \prime}(\dot{\varepsilon}, T)}, \\
& \tau_{d e b}^{\alpha}(\dot{\varepsilon}, T)=k_{d e b} \mu^{\alpha}(T) b^{\alpha} \sqrt{\rho_{d e b}(\dot{\varepsilon}, T)} \log \left(\frac{1}{b^{\alpha} \sqrt{\rho_{d e b}(\dot{\varepsilon}, T)}}\right),
\end{aligned}
$$

where $\chi^{S S \prime}$ is a dislocation-interaction matrix and $b^{\alpha}$ is the value of the Burgers vector. Here, only the diagonal terms $\left(s=s^{\prime}\right)$ are non-zero and assigned a typical value of 0.81 [40]. The evolution of $\rho_{\text {for }}^{S}(\dot{\varepsilon}, T)$ is goverend by a balance between the rate of dislocation storage and the rate of dislocation recovery following the theory on the thermodynamics of slip [40, 42, 43]:

$$
\frac{\partial \rho_{\text {for }}^{s}}{\partial \gamma^{s}}=\frac{\partial \rho_{\text {gen, for }}^{s}}{\partial \gamma^{s}}-\frac{\partial \rho_{\text {rem, for }}^{s}}{\partial \gamma^{s}}=k_{1}^{s} \sqrt{\rho_{\text {for }}^{s}}-k_{2}^{s}(\dot{\varepsilon}, T) \rho_{\text {for }}^{s},
$$

where $k_{1}^{s}$ is a rate-insensitive coefficient for dislocation storage by statistical trapping of dislocations, and $k_{2}^{s}$ is a rate-sensitive coefficient for dynamic recovery given by

$$
\frac{k_{2}^{s}}{k_{1}^{s}}=\frac{1}{\sqrt{\left(\chi^{-1}\right)^{s s^{\prime}} \frac{\tau_{s a t}^{\prime}}{b^{\alpha^{\prime}} \mu^{\alpha^{\prime}}}}}, \tau_{s a t}^{S}=\frac{D^{\alpha}\left(b^{\alpha}\right)^{3} g^{\alpha} \mu^{\alpha}}{D^{\alpha}\left(b^{\alpha}\right)^{3}-k T \log \left(\frac{\dot{\varepsilon}}{\varepsilon_{0}}\right)},
$$


In Eq. (7), $k, \dot{\varepsilon}_{0}, g^{\alpha}$, and $D^{\alpha}$ are, respectively, Boltzmann's constant, a reference strain rate, an effective activation enthalpy, and a dislocation drag stress.

The rate of debris $\rho_{d e b}(\dot{\varepsilon}, T)$ accumulation is related to the rate of recovery via:

$$
d \rho_{d e b}=q^{\alpha} b^{\alpha} \sqrt{\rho_{d e b}} \frac{\partial \rho_{r e m, f o r}^{s}}{\partial \gamma^{s}}\left|d \gamma^{s}\right|
$$

where $q^{\alpha}$ is a rate coefficient, related to the fraction of recovered dislocations that is stored as substructure.

When twins form within the grain, the twin boundaries can act as obstacles to slip. Accordingly, the term $\tau_{H P}^{S}$ is added in Eq. (11) representing the reduction in the effective grain size introduced by twin boundaries, as follows:

$$
\tau_{c}^{S}=\tau_{0}^{\alpha}+\tau_{\text {for }}^{S}+\tau_{d e b}^{\alpha}+\tau_{H P}^{S}
$$

where

$$
\tau_{H P}^{S}=\left(\frac{f^{p t s}-f^{p t s, 0}}{f^{p t s, m a x}-f^{p t s, 0}}\right)^{0.1} \mu^{\alpha}(T) H P^{\alpha} \sqrt{\frac{b^{\alpha}}{d_{m f p}^{S}}}
$$

and the constants $f^{p t s, 0}, f^{p t s, \max }$ representing the minimum and the maximum volume fraction that twin can occupy in a given grain are taken to be 0.05 and 0.95 , respectively. This term is known as the barrier factor. The bigger the twin the larger barrier it presents for moving dislocations. $H P^{\alpha}$ is the Hall-Petch coefficient, $\mu^{\alpha}(T)$ is the shear modulus as a function of temperature $T$, and $d_{m f p}^{S}$ is the mean free path given by [33, 35]:

$$
d_{m f p}^{s}=\frac{\left(1-f^{p t s}\right) d_{c}}{\sin (\theta)}
$$

where the angle $\theta$ is defined between the slip plane of system $s$ and the twin plane of the twin system.

The threshold stresses for activating a given twin system (variant) $t$ within a given twin mode $\beta$ is modeled as a sum of two resistances:

$$
\tau_{c}^{\beta}=\tau_{0}^{\beta}+\tau_{\text {slip }}^{\beta}
$$

where $\tau_{0}^{\beta}$ is a temperature-independent friction term and $\tau_{\text {slip }}^{\beta}$ is a latent hardening term given by

$$
\tau_{\text {slip }}^{\beta}=\sum_{s} C^{\alpha \beta} b^{\beta} \mu^{\alpha}(T) b^{\alpha} \rho_{\text {for }}^{s}
$$


where $C^{\alpha \beta}$ is the latent hardening matrix. This term represents the resistance to twin expansion provided by the stored dislocation density within the same grain.

\subsection{Material model for alpha-uranium}

The predominant slip and twinning modes used in the plastic deformation of $\alpha$-U have been identified in previous works $[2,3,20,44-51]$. The crystallographic plane and direction and the number of independent systems of these modes are given in Fig. 2. The (010)[100] slip mode (called wall slip) is the easiest to activate at room temperature [1,3]. Another relatively easy slip mode is floor slip (001)[100], and like wall slip, also possesses only one slip system. The $1 / 2\{110\}\langle 1 \overline{1} 0\rangle$ slip mode, referred to as chimney slip, has two slip systems, and is often found to be the next easiest to activate. The $1 / 2\{1 \overline{1} 2\}\langle 021\rangle$ slip mode, or roof slip, is relatively harder, but unlike the previous three modes accommodates plastic strain in the [001] direction. The main twinning modes reported in experimental studies of deformed uranium are: $\{130\}\langle 3 \overline{1} 0\rangle,\{172\}\langle 3 \overline{1} 2\rangle$ and $\{112\}\langle 3 \overline{7} 2\rangle[2,3,44]$. The first one, the $\{130\}\langle 3 \overline{1} 0\rangle$ twin, is the most frequently oberved and its twin shear $S$ is 0.299 and it reorients the lattice by $69.3^{\circ}$ about [001].

\begin{tabular}{|c|c|c|c|c|c|}
\hline $\begin{array}{c}\text { Wall slip } \\
(010)[100] \\
1 \text { Slip System } \\
2.85 \times 10^{-10}\end{array}$ & $\begin{array}{l}\text { Floor slip } \\
(001)[100] \\
1 \text { Slip System } \\
2.85 \times 10^{-10}\end{array}$ & $\begin{array}{c}\text { Chimney slip } \\
\frac{1}{2}\{110\}\langle 1 \overline{1} 0\rangle \\
2 \text { Slip Systems } \\
6.51 \times 10^{-10}\end{array}$ & $\begin{array}{c}\text { Roof slip } \\
\frac{1}{2}\{021\}\langle 1 \overline{1} 2\rangle \\
4 \text { Slip Systems } \\
11.85 \times 10^{-10}\end{array}$ & $\begin{array}{c}\{130\}\langle 3 \overline{1} 0\rangle \\
\text { twinning } \\
2 \text { Twin Variants } \\
10.36 \times 10^{-11}\end{array}$ & $\begin{array}{c}\{172\}\langle 3 \overline{1} 2\rangle \\
\text { twinning } \\
4 \text { Twin Variants } \\
14.33 \times 10^{-10}\end{array}$ \\
\hline & & & & & \\
\hline
\end{tabular}

Fig. 2. Deformation modes in $\alpha$-uranium. The arrows indicate the Burgers vector in the planes. The magnitude of the Burgers vector is also given in meters $\left(b^{\alpha}[\mathrm{m}]\right)$.

These predominant slip and twinning systems are made available in the $\alpha$-U deformation simulations to follow. The material parameters corresponding to these modes have been calibrated in prior work [52] and are given in Tables I and II for slip and twinning, respectively. Consistent with conventional thought and prior modeling work [4], the initial athermal activation 
stress for (010)[100] slip is the lowest, (001)[100] slip the second lowest, $1 / 2\{110\}\langle 1 \overline{1} 0\rangle$ slip larger, and $1 / 2\{021\}\langle 1 \overline{1} 2\rangle$ the largest.

Table I. Model parameters for the evolution of slip system resistance.

\begin{tabular}{lcccc}
\hline & Wall slip & Chimney slip & Floor slip & Roof slip \\
& $\alpha=1,(010)[100]$ & $\alpha=2,1 / 2\{110\}\langle 1 \overline{1} 0\rangle$ & $\alpha=3,(001)[100]$ & $\alpha=4,1 / 2\{021\}\langle 1 \overline{1} 2\rangle$ \\
\hline$H P^{\alpha=1,2}$ & 50.0 & 50.0 & 300.0 & 50.0 \\
$k_{1}^{\alpha}\left[m^{-1}\right]$ & $1.2 \times 10^{8}$ & $2.0 \times 10^{7}$ & $1.0 \times 10^{7}$ & $2.2 \times 10^{8}$ \\
$g^{\alpha}$ & 0.01 & 0.01 & 0.01 & 0.01 \\
$D^{\alpha}[\mathrm{MPa}]$ & 446 & 70 & 126 & 126 \\
$q^{\alpha}$ & 18.0 & 4.0 & 0.0 & 80.0 \\
\hline \multicolumn{1}{c}{$\mathrm{A}$} & 255 & 655 & 590 & 900 \\
$\mathrm{~B}$ & 380 & 140 & 350 & 940
\end{tabular}

Table II. Model parameters for the evolution of twin resistance.

\begin{tabular}{ccc} 
& $\beta=1,\{130\}\langle 3 \overline{1} 0\rangle$ & $\beta=1,\{172\}\langle 3 \overline{1} 2\rangle$ \\
\hline$\tau_{0}^{\beta}$ & 100.0 & 500.0 \\
$C^{1 \beta}$ & 5500 & 5500 \\
$C^{2 \beta}$ & 5500 & 5500 \\
$C^{3 \beta}$ & 8500 & 8500 \\
$C^{4 \beta}$ & 6800 & 6100
\end{tabular}

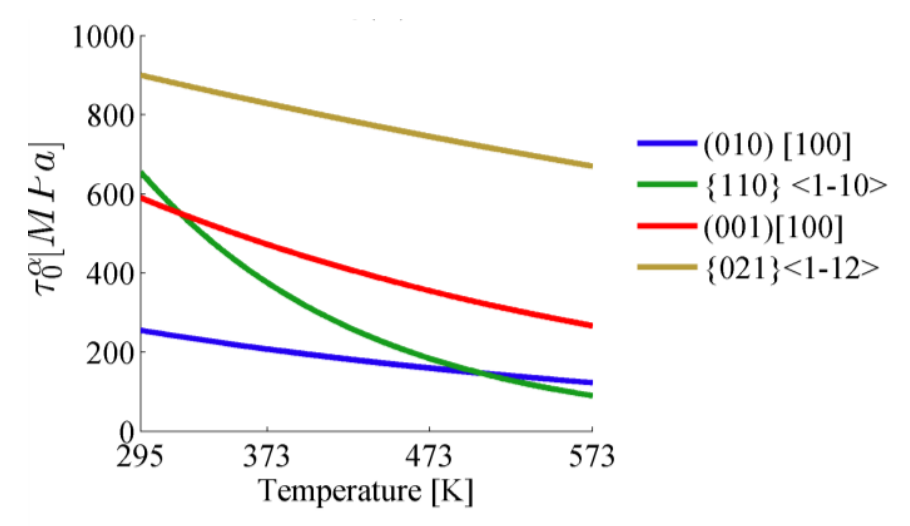

Fig. 3. Predicted dependence of the initial slip resistance on temperature for each slip mode [52].

\subsection{Model set up for deformation simulations}

The $\alpha-\mathrm{U}$ material under investigation has been deformed at room temperature and $573 \mathrm{~K}$ in simple compression in three directions and rolling, respectively [5]. Both types of deformation, rolling and simple compression, are simulated and the evolution of bulk texture and the evolution of different texture components are analyzed. For rolling, we impose homogeneous boundary 
conditions corresponding to (1) plane strain compression (PSC). PSC is an idealization of rolling that applies best near the center of the rolled sheet [53-55]. It does not account for any possible alterations in deformation caused by the interactions between the rolls and the sample at the two surfaces of the sheet. For simple compression (SC), we impose the prescribed load direction with respect to the initial texture and traction-free surfaces in the other two orthogonal directions.

In all calculations, the initial texture was assigned using the measured data in Fig. 1a and the strain rate was set to $0.001 / \mathrm{s}$. The temperature ranged from room temperature to $573 \mathrm{~K}$. The TT direction of the initial texture corresponds to the normal direction (ND), while the IP1 and IP2 directions correspond to the transverse (TD) and rolling direction (RD), respectively.

\section{Results}

\subsection{Texture evolution during plane strain compression}

The texture evolution during PSC was simulated at both room temperature and $573 \mathrm{~K}$ from $16 \%$ to $60 \%$ reduction. Figures $4 \mathrm{a}$ and c show the development at room temperature and $573 \mathrm{~K}$ respectively. The model predicts that beyond 16\% strain, texture evolution depends on temperature. At room temperature, four $\{001\}$ peaks develop as strain increases. In contrast, at $573 \mathrm{~K}$, the texture develops two peaks near the TT in the $\{001\}$ pole figure, a small peak in the $\{010\}$ pole figure in the IP2 direction and a $\{100\}$ peak in the IP1 direction. The two peaks in the $\{001\}$ lie approximately at $\pm 10-15^{\circ}$ from the IP1-TT plane.

Uranium is not commonly rolled at room temperature but rather at $573 \mathrm{~K}$ and above. For validation, we provide in Fig. 4b the measured EBSD textures for the same rolling reductions at $573 \mathrm{~K}$. Overall the agreement is good, particularly in capturing the development of the $\{001\}$ peaks. The slight asymmetry in the intensity of these peaks found in the calculated texture arises from the asymmetry in the initial texture and the grain co-rotation scheme.

The development of texture at $573 \mathrm{~K}$ involves both slip and twinning. As another form of validation, we compare the predicted and measured twin volume fractions in Fig. 5. As rolling progresses, the twin fraction increases to nearly 30\%. As shown, the calculated twin fractions agree well with the measured fractions.

Differences in texture development at room and high temperature seen in Fig. 4 are a result of differences in the slip and twinning systems activated. Figure 6 presents the slip and twin activities corresponding to rolling at these two temperatures. We find that rolling deformation at 
both temperatures involves multiple slip and twinning on the $\{130\}\langle 3 \overline{1} 0\rangle$ systems and no twinning on the $\{172\}\langle 3 \overline{1} 2\rangle$ systems. The main differences are that $\{110\}\langle 1 \overline{1} 0\rangle$ slip is less active and $\{021\}\langle 1 \overline{1} 2\rangle$ slip is more activate at room temperature than at high temperature.

It is worth noting a few interesting parallels between the rolling textures of $\alpha-\mathrm{U}$, which has a c/a ratio of 1.734 and $\mathrm{HCP}$ metals with similarly high c/a ratios, like $\mathrm{Zn}$ (1.856) and $\mathrm{Cd}$ (1.885). $\mathrm{Zn}$ and $\mathrm{Cd}$ tend to exhibit textures with their basal poles tilted $\pm 15-25^{\circ}$ away from the TT-TD plane toward RD (IP2 in our case) [56-58]. This type of texture development results from a dominance of basal slip [56, 58, 59]. Floor slip in the orthorhombic unit cell of $\alpha-U$ is geometrically similar to basal slip in the hexagonal close packed (HCP) unit cell. Here we see that predominance in floor slip has led to the development of pronounced peaks $\pm 10-15^{\circ}$ away from the TT toward IP2.

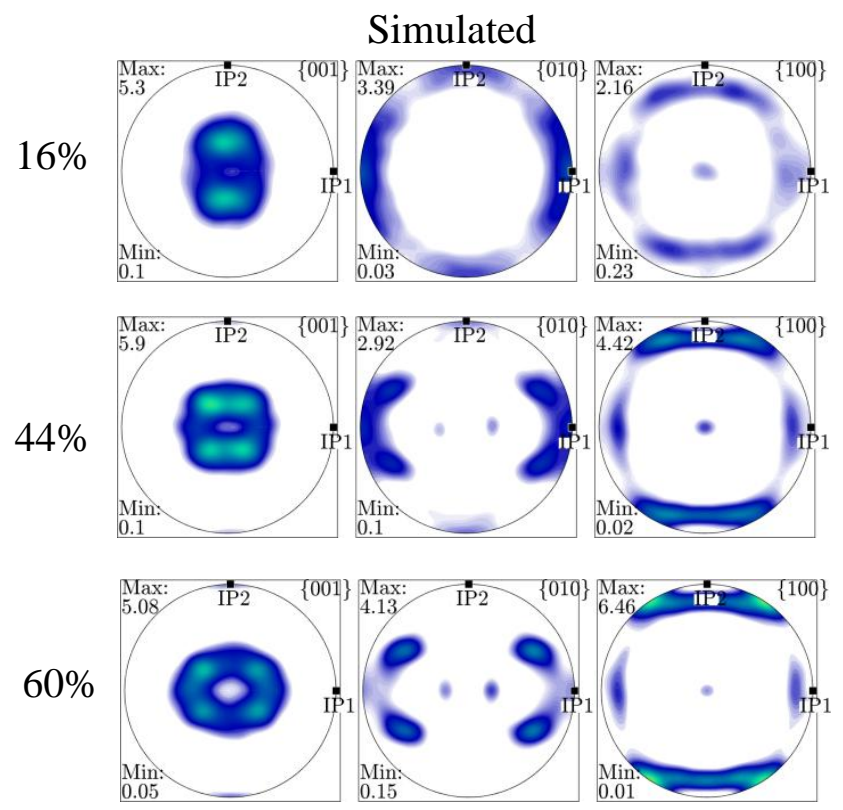


$573 \mathrm{~K}$

Reduction Measured
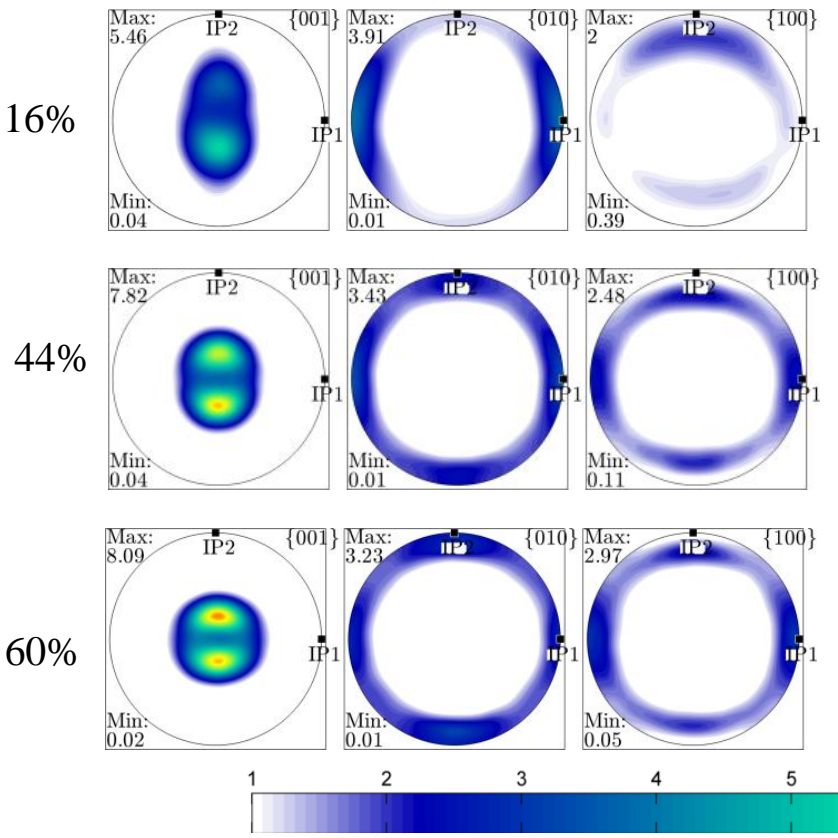

Simulated
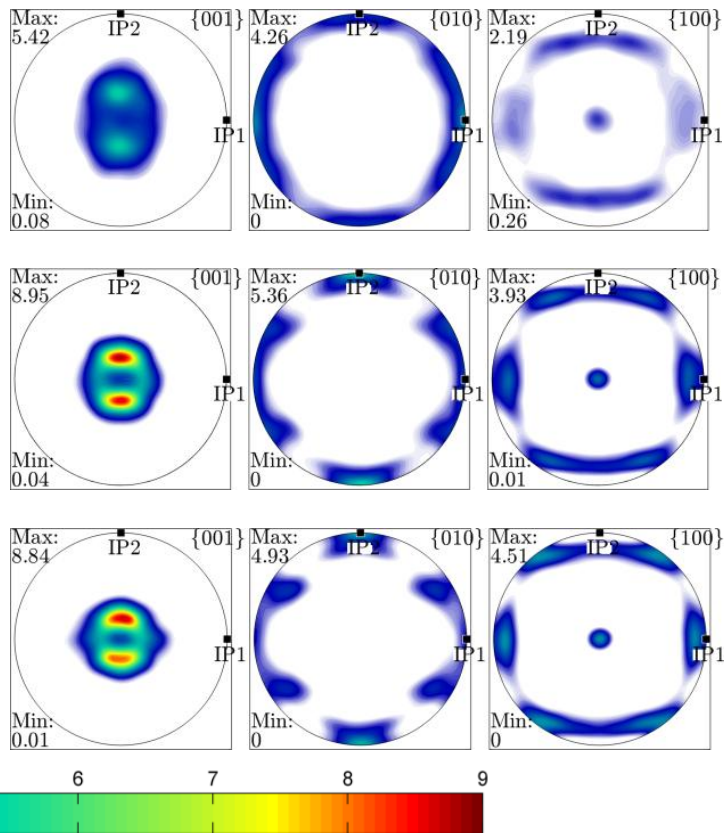

Fig. 4. Comparison of measured and predicted texture evolution during plane strain compression (PSC) of $\alpha-\mathrm{U}$ at room temperature and $573 \mathrm{~K}$. The PSC reductions correspond to the following true strain: $-0.174,-0.58$, and -0.916 .

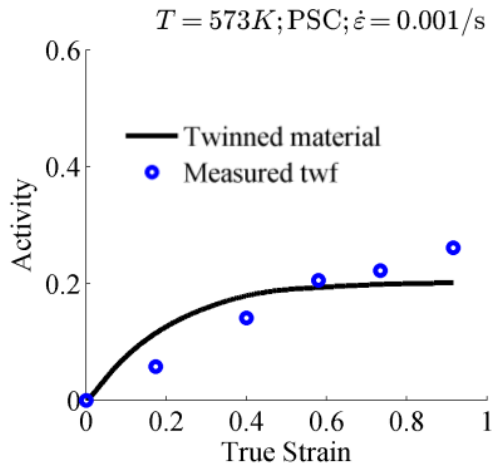

Fig. 5. Comparison of the twin volume fraction evolution between the model and experiment during plane strain compression at $573 \mathrm{~K}$. 
$295 \mathrm{~K}$
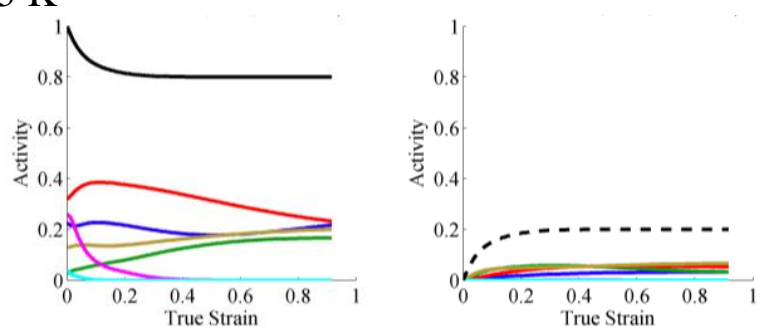

$573 \mathrm{~K}$
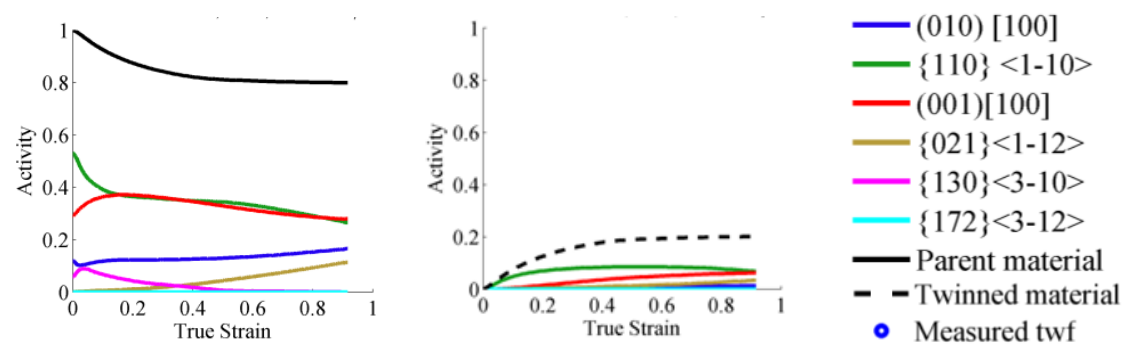

Fig. 6. Predicted relative activities of active deformation modes within parent grains (left) and within twins (right) during plane strain compression at room temperature (top row) and $573 \mathrm{~K}$ (bottom row). Also plotted are the corresponding parent material and twinned material volume fraction.

\subsubsection{Lattice reorientations due to slip}

In the foregoing section, we have seen substantial evolution of texture in plane strain compression of uranium and the analysis from polycrystal model indicates that it results from the activity of multiple slip and twinning modes. To obtain a deeper understanding of texture development, we use the same polycrystalline plasticity VPSC model with the aim of uncovering the origin of the observed crystal reorientations involved in texture evolution and the activated slip modes in $\alpha-U$. For reasons we discuss shortly, we will also engage results from a second polycrystal model, a Sachs-like model. Both models are used to calculate the reorientation tendencies (averaged over all grains) observed for a given slip mode and twinning mode during plane strain compression. The prior analysis indicates that the prominent deformation modes are: Wall slip (010)[100], Chimney slip $1 / 2\{110\}\langle 1 \overline{1} 0\rangle$, Floor slip (001)[100], Roof slip $1 / 2\{021\}\langle 1 \overline{1} 2\rangle$, and $\{130\}\langle 3 \overline{1} 0\rangle$ and $\{172\}\langle 3 \overline{1} 2\rangle$ twinning. For the VPSC analysis, the initial texture is the texture taken from the measurement in Fig. 1a and for the Sachs-like model analysis a random texture represented by 1000 orientations in Fig. 1 b.

Figure 7 shows the direction and magnitudes of lattice reorientation of plane normals projected in three pole figures at a strain of 0.001 from the VPSC calculation. The reorientation velocity per crystal is calculated using the following procedure: (1) the initial and reoriented 
crystal orientations are recorded, (2) the selected initial and reoriented plane normals (e.g., (001)) are projected using the equal area projection in a pole figure as two points, and (3) the two points are connected by an arrow pointing from the starting point towards the reoriented point. Since the deformation step is very small we can assume that a vector connecting these two points on a unit sphere lies in the direction of the reorientation velocity. The magnitude of this velocity is scaled by an arbitrary constant for each slip mode to fit the arrows within a pole. Therefore absolute reorientation magnitudes should not be compared between different modes. Furthermore, for several poles, velocity plots were averaged over certain areas so that the reorientation patterns could be better revealed. To this end, the velocity field within a certain area was summed and then divided by the corresponding area fraction.

With deformation in VPSC, the crystals reorient and the resistances to slip evolve differently for each grain. These changes alter the relative activity of deformation modes. Because of this, we repeated the analysis presented in Fig. 7 for certain slip modes at additional amounts of strain. Figure 8 shows the lattice reorientation velocity at a compression strain of 0.8 for roof slip and at a strain of 0.8 for chimney slip. The roof slip is much more active at high strains and its role can be better revealed at higher strains. It is acting against floor slip and spreading the peaks on $\{001\}$ pole figure while relaxing the IP2 peak in the $\{010\}$ pole. Also, the role of chimney slip is clearer at higher strains.

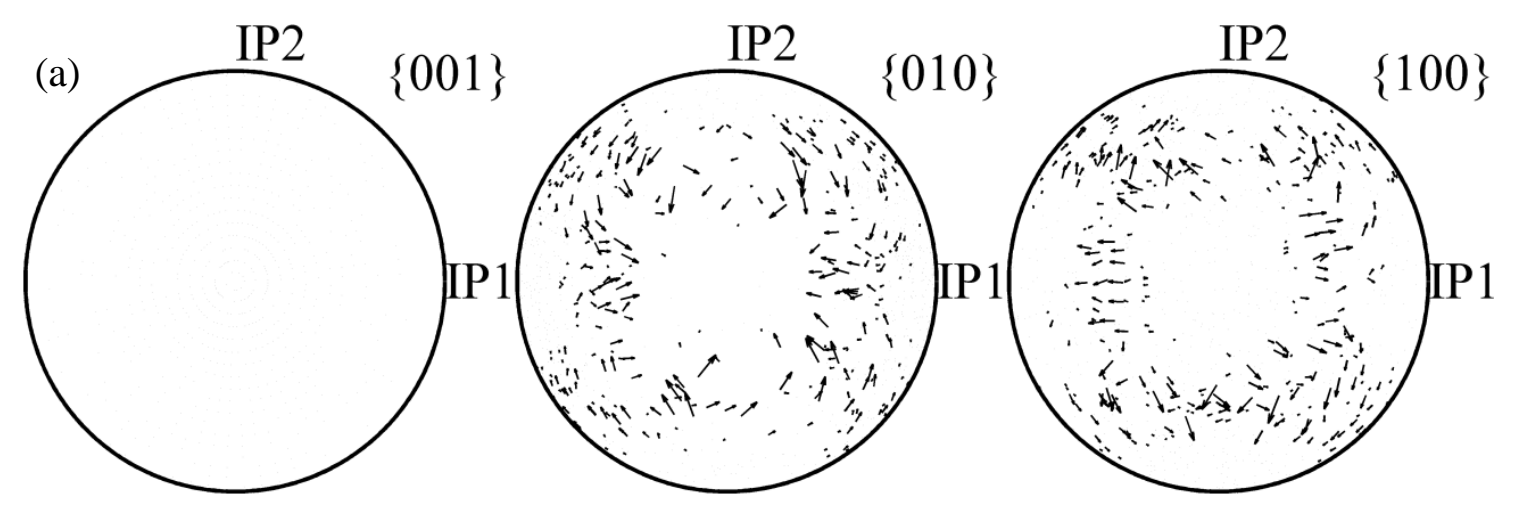




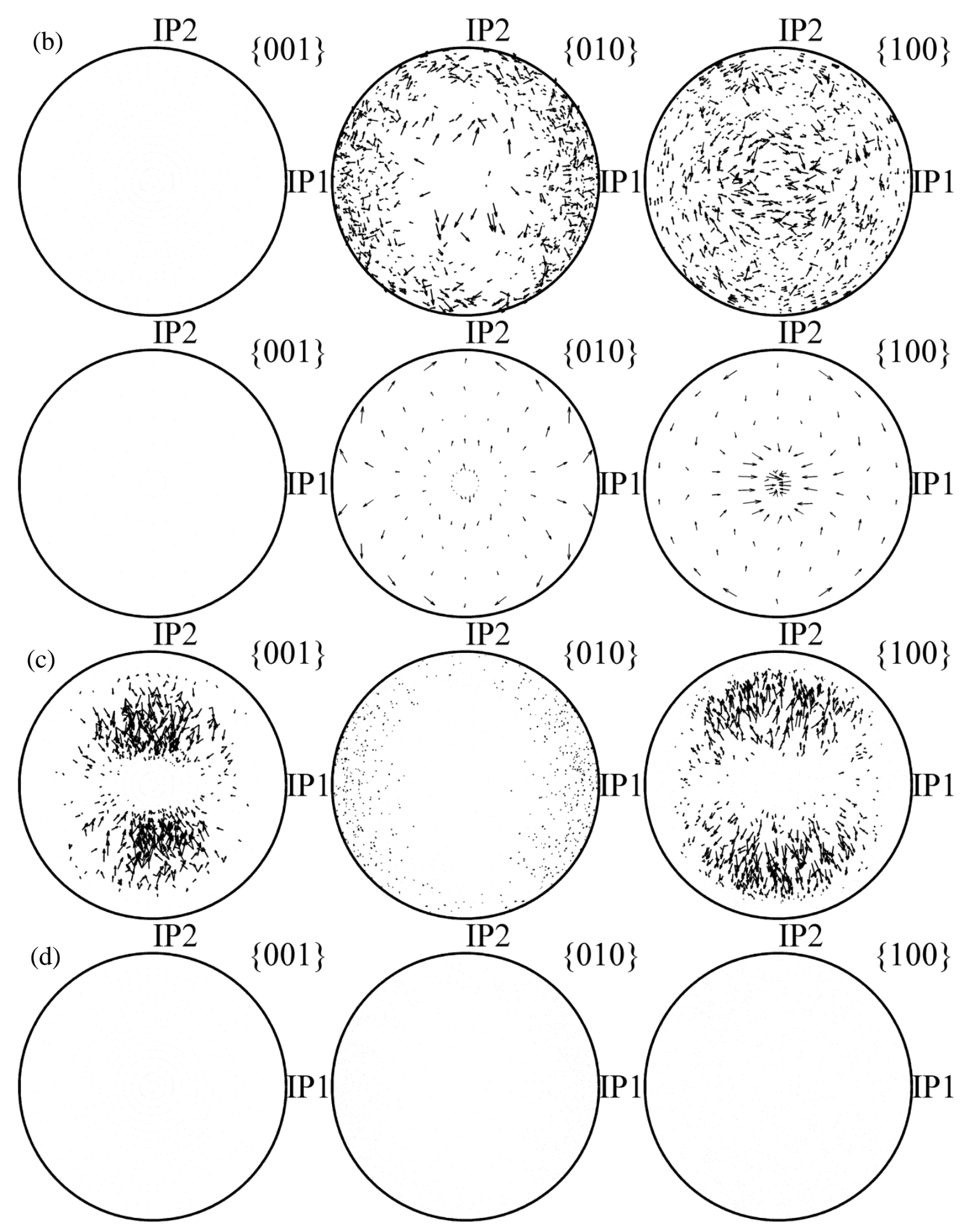

Fig. 7. Direction and magnitude of lattice orientation under plane strain compression in the through-thickness direction at $573 \mathrm{~K}$ to a strain of 0.001 predicted by the VPSC model for each slip mode. The calculations correspond to the relative fraction of deformation accommodated by (a) wall slip, (b) chimney slip (along with averaged poles for clarity), (c) floor slip and (d) roof slip shown in Fig. 6 and the SR initial texture. Roof slip is inactive and therefore (d) appears empty. 
(a)

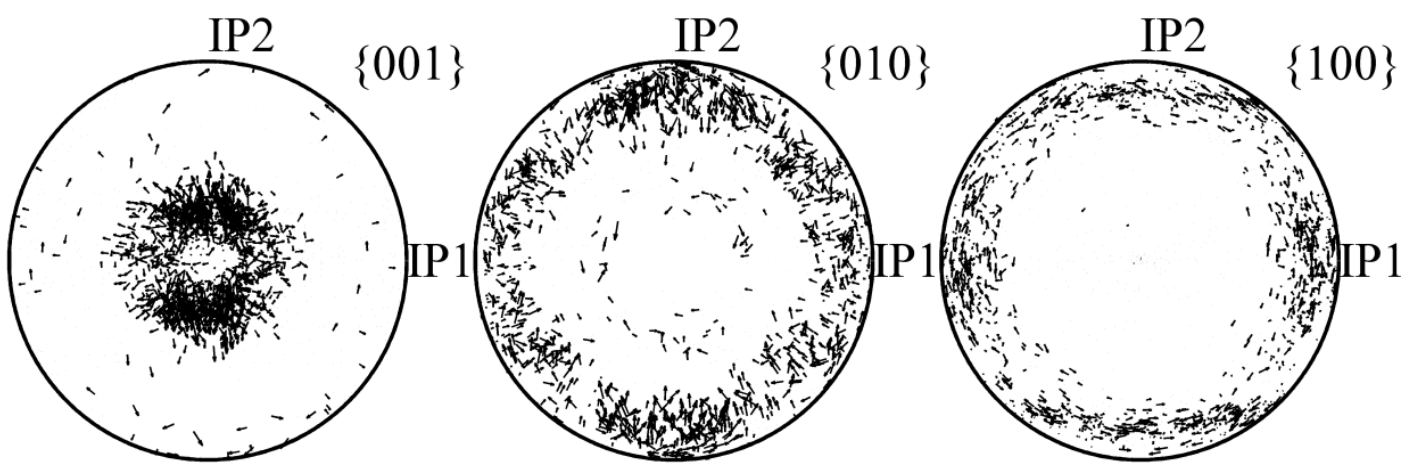

(b)
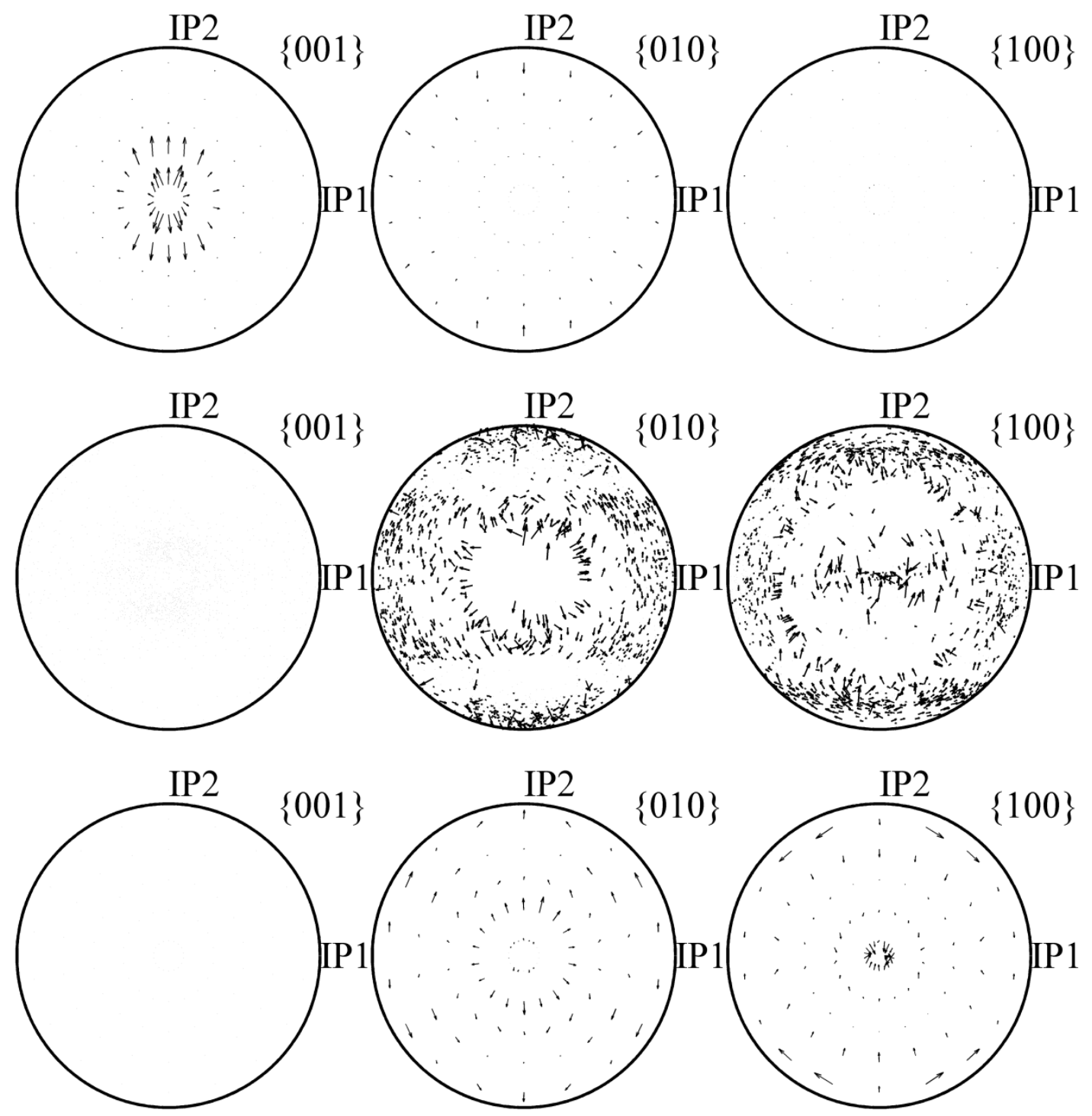

Fig. 8. Direction and magnitude of lattice orientation under plane strain compression in the through-thickness direction at $573 \mathrm{~K}$ at a strain of 0.8 predicted by the VPSC model: (a) roof slip 
reorientation at 0.8 compressive strain along ND (the averaged poles are also provided for clarity); (b) chimney slip reorientation at 0.8 compressive strain along ND (the averaged poles are also provided for clarity).

In the VPSC calculation, when plane strain boundary conditions are applied, crystals favorably oriented to accommodate the applied deformation will predominantly deform while crystals not favorably oriented will deform very little or not at all. Further, each mode will not operate alone in the crystal and, thus, the reorientation tendencies will be based on the relative activities of multiple deformation modes. In the Sachs model, however, it is possible to enforce a single deformation mode to accommodate the entire deformation. Unlike VPSC model, a Sachs model can reveal the lattice reorientations of the inactive deformation modes. For example, this explains why the pole figures calculated from VPSC in Fig. 7d are empty. To eliminate this deficiency and enforce accommodation of an applied deformation with only one active slip mode in all grains, we repeat the lattice reorientation calculations using a Sachs-like model. Although these calculations may be less representative of the actual texture evolution seen experimentally, we believe it is worthwhile to confirm reorientation tendencies caused by each mode in isolation.

In the classic Sachs model, the stress is kept the same in each crystal while the strain is allowed to vary from crystal to crystal. To approach as close as possible to the strain rate boundary conditions for PSC while at the same time maintaining the uniform stress tensor across crystals, we utilize the following capability of the VPSC model. The VPSC model can be carried out with a fully constrained (the Taylor type [60-65]) and several grain-effective medium interaction linearization procedures. The latter covers a range from the stiff secant $n^{e f f}=1$ to the compliant tangent $n^{e f f}=n$ linearizations. Intermediate options, such as the affine and the $n^{\text {eff }}=10$ interaction schemes, are also available and more often used [66]. These intermediate linearization procedures are approximations that give responses of a polycrystalline material inbetween the Taylor upper bound model [60, 67-70] (or closer to the stiff secant approach) and the Sachs lower bound model $[71,72]$ (or closer to the compliant tangent approach). By setting the $n^{e f f}$ parameter to a large value, the matrix becomes compliant and the response is very close to that calculated by the Sachs model [73]. In fact, $n^{\text {eff }}=500$ closely approximates the uniform grain stress condition under plane strain. For the Sachs calculations, we considered a polycrystal with no initial texture (random) represented by 1000 discrete orientations, (Fig. 1b), in order to eliminate the effect of preferred orientation and ensure uniform coverage of pole figures. Results 
are presented in Fig. 9. In general, results predicted by the Sachs model are similar to those predicted by the VPSC model with a benefit that the role of roof slip can be observed.

We note that wall slip does not cause any reorientation on the pole (001), which is reasonable since the angular velocity caused by the plastic spin will always be perpendicular to slip plane normal and slip direction. This can be seen when considering the plastic spin arising from the shear provided by only one system:

$$
W_{i j}^{p}=\frac{1}{2} \dot{\gamma}\left(b_{i} n_{j}-n_{i} b_{j}\right)
$$

where $b_{i}$ and $n_{j}$ represent components of the slip direction and slip plane normal. The dual vector representing the corresponding angular velocity acting on crystal is:

$\boldsymbol{\omega}^{p}=\omega_{k}^{p} \mathbf{e}_{k}=-\frac{1}{2} \varepsilon_{i j k} W_{i j}^{p} \mathbf{e}_{k}=-\frac{1}{4} \dot{\gamma}\left(\varepsilon_{i j k} b_{i} n_{j} \mathbf{e}_{k}-\varepsilon_{i j k} n_{i} b_{j} \mathbf{e}_{k}\right)=-\frac{1}{4} \dot{\gamma}(\mathbf{b} \times \mathbf{n}-\mathbf{n} \times \mathbf{b})=$

$-\frac{1}{2} \dot{\gamma} \mathbf{b} \times \mathbf{n}$

Therefore, for the (010)[100] mode, the direction of angular velocity will be in (001) and thus causes no reorientation in the (001) pole figure. The same applies for the (001)[100] mode, which causes no reorientation in the (010) pole figure. The most active grains are those with slip plane normal and slip direction oriented $\pm 45^{\circ}$ with respect to the TT in the TT-IP1 plane. This situation applies to all modes and can be clearly seen on reorientation plots for the (010)[100] and (001)[100] slip modes.

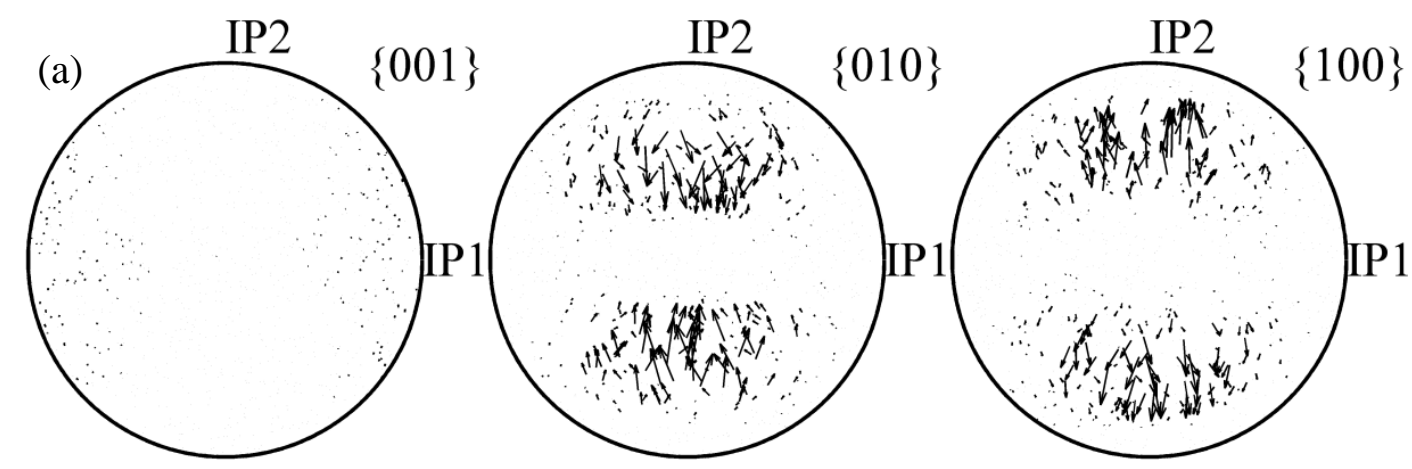




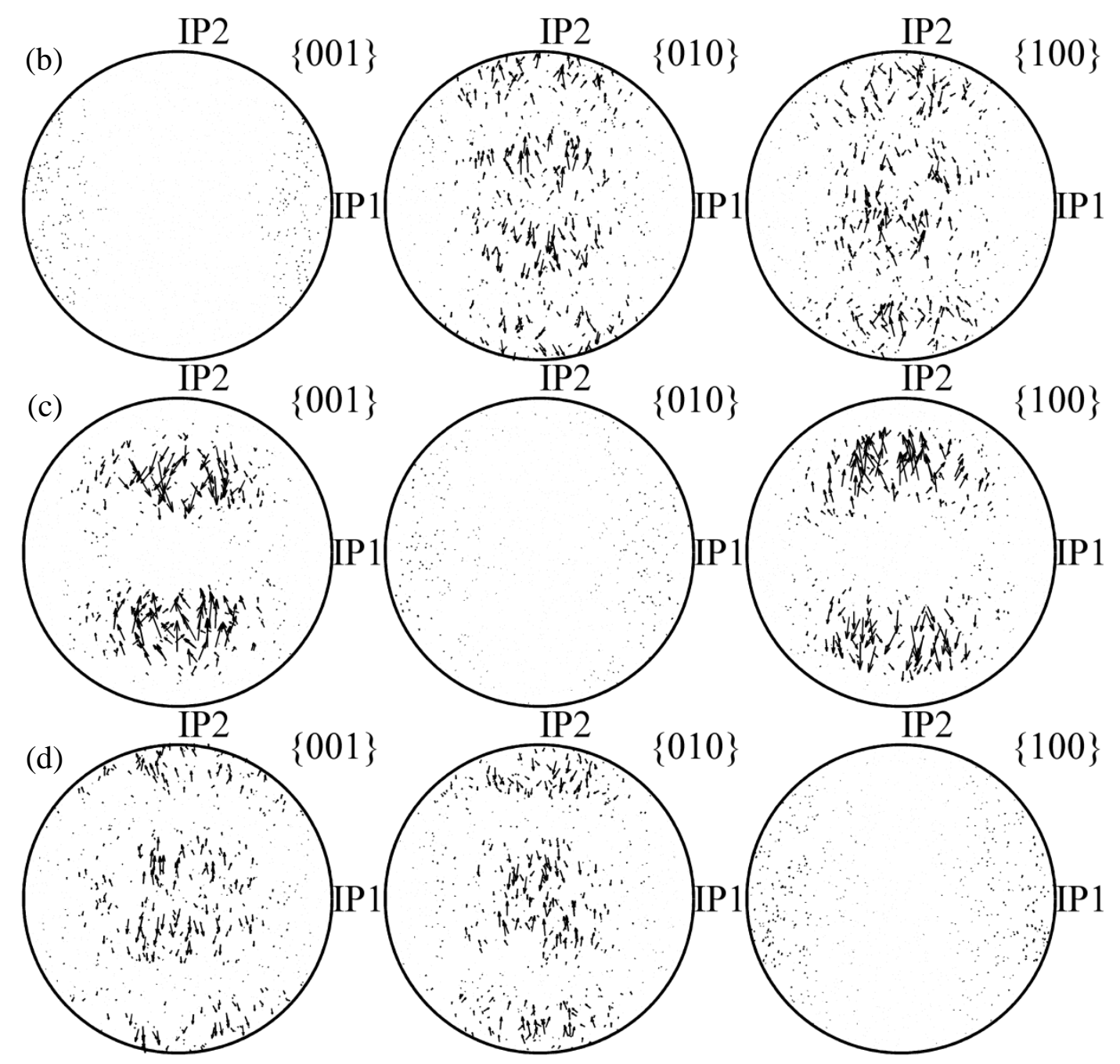

Fig. 9. Direction and magnitude of lattice reorientation under plane strain compression in the through-thickness direction at $573 \mathrm{~K}$ to a strain of 0.001 predicted by the Sachs model when the entire deformation is accommodated by a single slip mode either (a) wall slip, (b) chimney slip, (c) floor slip or (d) roof slip. The initial texture was random and represented using 1000 orientations.

\subsubsection{Lattice reorientations due to twinning}

Deformation twinning causes significant and abrupt lattice reorientations compared to slip. As mentioned earlier, the $\{130\}\langle 3 \overline{1} 0\rangle$ deformation twins predominate in $\alpha$-U over the $\{172\}\langle 3 \overline{1} 2\rangle$ twins. Thus, in our analysis of lattice reorientations from twinning, we use the VPSC model to study $\{130\}\langle 3 \overline{1} 0\rangle$ twinning only.

Figure 10 shows reorientation plots due to twinning during plane strain compression at two strain levels, 0.1 and 0.4 , and for two starting textures: the initially textured material in Fig. 1a and the initially random texture in Fig. 1b. The blue points correspond to grain orientations that 
are more likely to twin and the red points show the orientations of their twins. The analysis indicates that the initial texture, more so than the strain level, has an influence on which grains have a propensity for twinning.

(a) $-0.1$

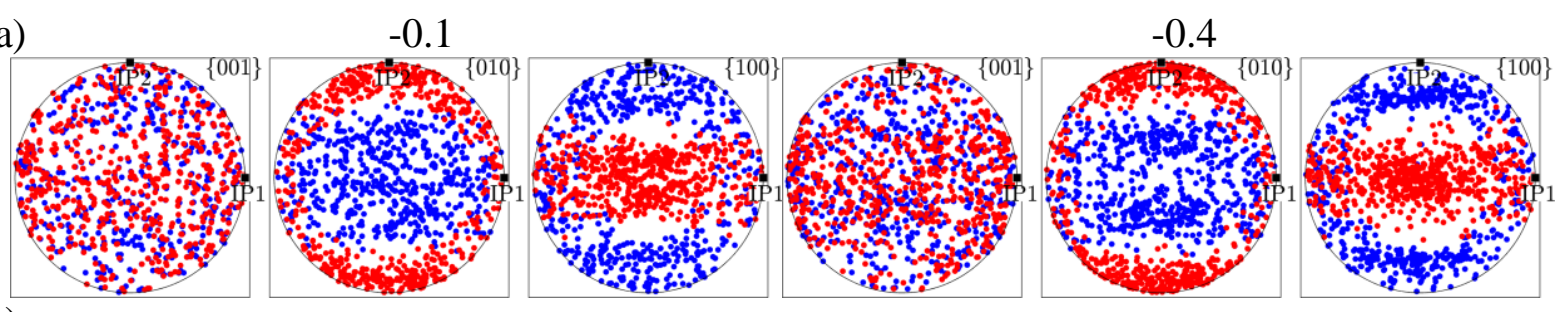

(b)

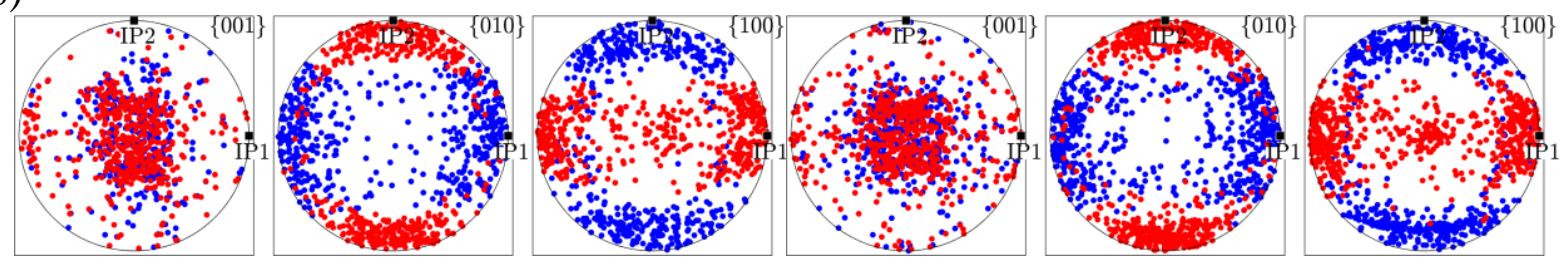

Fig. 10. Parent grains that develop twins (blue) and their twins (red) after 0.1 and 0.4 plane strain compression along the through-thickness direction for (a) the random texture material (Fig. 1b) and (b) the initially textured material (Fig. 1a).

\subsubsection{Lattice reorientations tendencies}

Figure 11 summarizes the lattice reorientations associated with plane strain compression at $573 \mathrm{~K}$, the temperature used in rolling of $\alpha-\mathrm{U}$ in practice. The two distinct maxima on the $\{001\}$ pole are predominantly caused by (001)[100] floor slip. The floor slip, (001)[100], also forms upper and lower concentric areas in the $\{100\}$ pole. The two peaks in the $\{010\}$ pole figure in the IP2 direction result from $\{130\}\langle 3 \overline{1} 0\rangle$ twins. At the same time, reorientation of grains (parents) containing $\{130\}\langle 3 \overline{1} 0\rangle$ twins weakens the $\{010\}$ poles in the IP1 direction. Additionally, $\{130\}\langle 3 \overline{1} 0\rangle$ twins strengthen the $\{100\}$ pole in the IP1 and weaken it in IP2 direction. Slip reorients grains through continuous reorientation, and large activity of the chimney slip, $1 / 2\{110\}\langle 1 \overline{1} 0\rangle$, strengthens the IP2 peaks in the $\{010\}$ pole and the IP1 peaks in the $\{100\}$ pole. Finally, activity of the wall slip, (010)[100], has a similar effect on the $\{100\}$ pole as floor slip and causes, with the aid of chimney slip, formation of the texture components in the $\{010\}$ pole that are inclined from the TT-IP1 about $35^{\circ}$ towards IP2. Finally, at the later stages of deformation roof slip relaxes the IP2 peak in the $\{010\}$ pole and two peaks on the $\{001\}$. 
A similar tendency diagram is provided for plane strain compression at room temperature (Fig. 11b). For the most part, Fig. 11 for $573 \mathrm{~K}$ can explain the room temperature plane strain compression as well. Only the effect of roof slip is missing since the main difference between room temperature and $573 \mathrm{~K}$ plane strain compression is that roof slip and wall slip are considerably more active, and chimney slip is not active at room temperature. Thus, Fig. 11a is updated by adding the orientation tendency due to the roof slip and shown in Fig. 11b.

(a) $573 \mathrm{~K}$ PSC

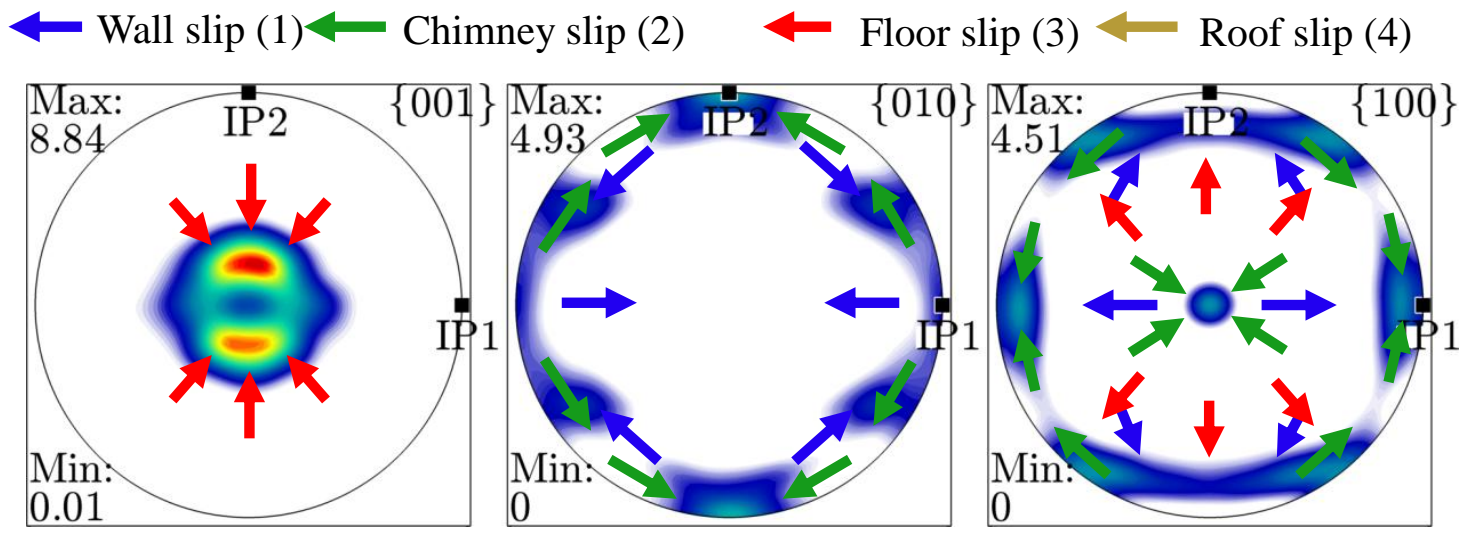

(b) $295 \mathrm{~K}$ PSC
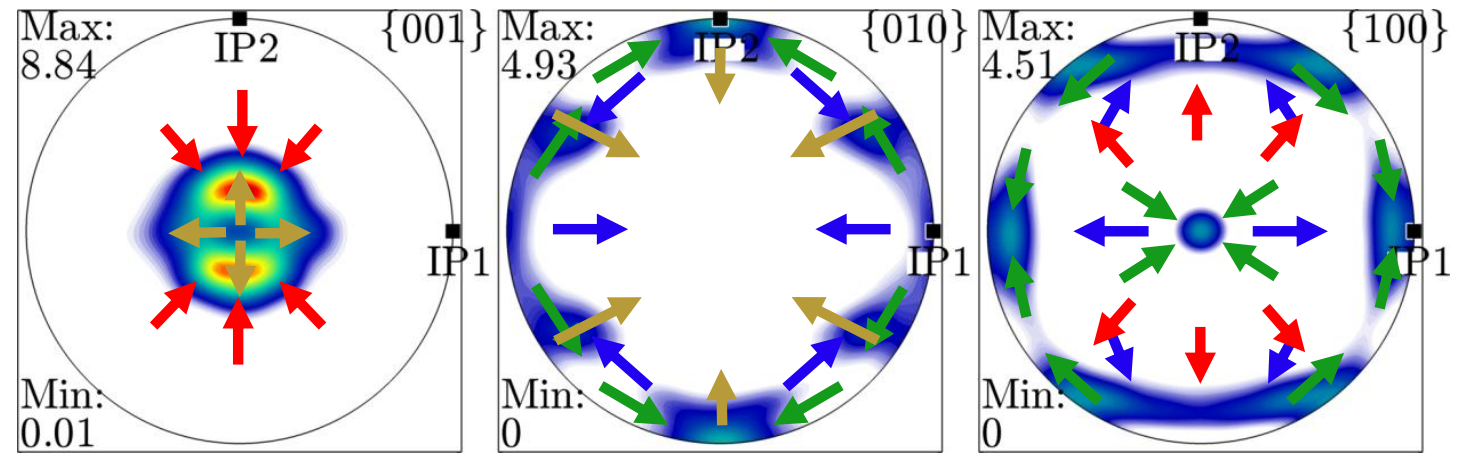

Fig. 11. Summary of lattice reorientations due to slip in plane strain compression. Figure 10 shows lattice reorientation due to twinning.

\subsection{Texture evolution during simple compression}

In this section, we examine texture evolution during room temperature simple compression in one of three orthogonal directions. Figure 12 compares the calculated texture evolution with that measured using EBSD. In general the texture evolution at room temperature is captured well by the model. 
Under deformation in all directions, the texture has evolved noticeably from the initial state to achieve a strong texture. The final texture after IPC1 is markedly different than that after compression in IPC2 and TTC3. In IPC1, the (001) poles concentrate along the TT direction, the (100) poles along IPC1, which is the direction of loading, and the (010) poles in the IP2 direction. In contrast, for IPC2, the (001) poles are distributed in the TT-IP2 plane. The (100) poles concentrate along the IP2 direction of loading and the (010) poles peak in the IP1 direction. In TTC3, the texture appears weaker than for the in-plane tests. We see the development of two maxima about $15-25^{\circ}$ from the TT direction towards the IP2 direction. There are diffuse and weak (100) peaks along the IP2 direction and diffuse and weak (010) peaks in the IP1 direction. The corresponding model predictions are also provided in these figures for validation.

Room temperature compression in all three directions involved twinning on the $\{130\}\langle 3 \overline{1} 0\rangle$ systems. As a form of validation, Fig. 13 compares the measured twin fractions at the end of deformation with the evolution in twin fraction as calculated by the model. The model predicts the amount of twinning well.

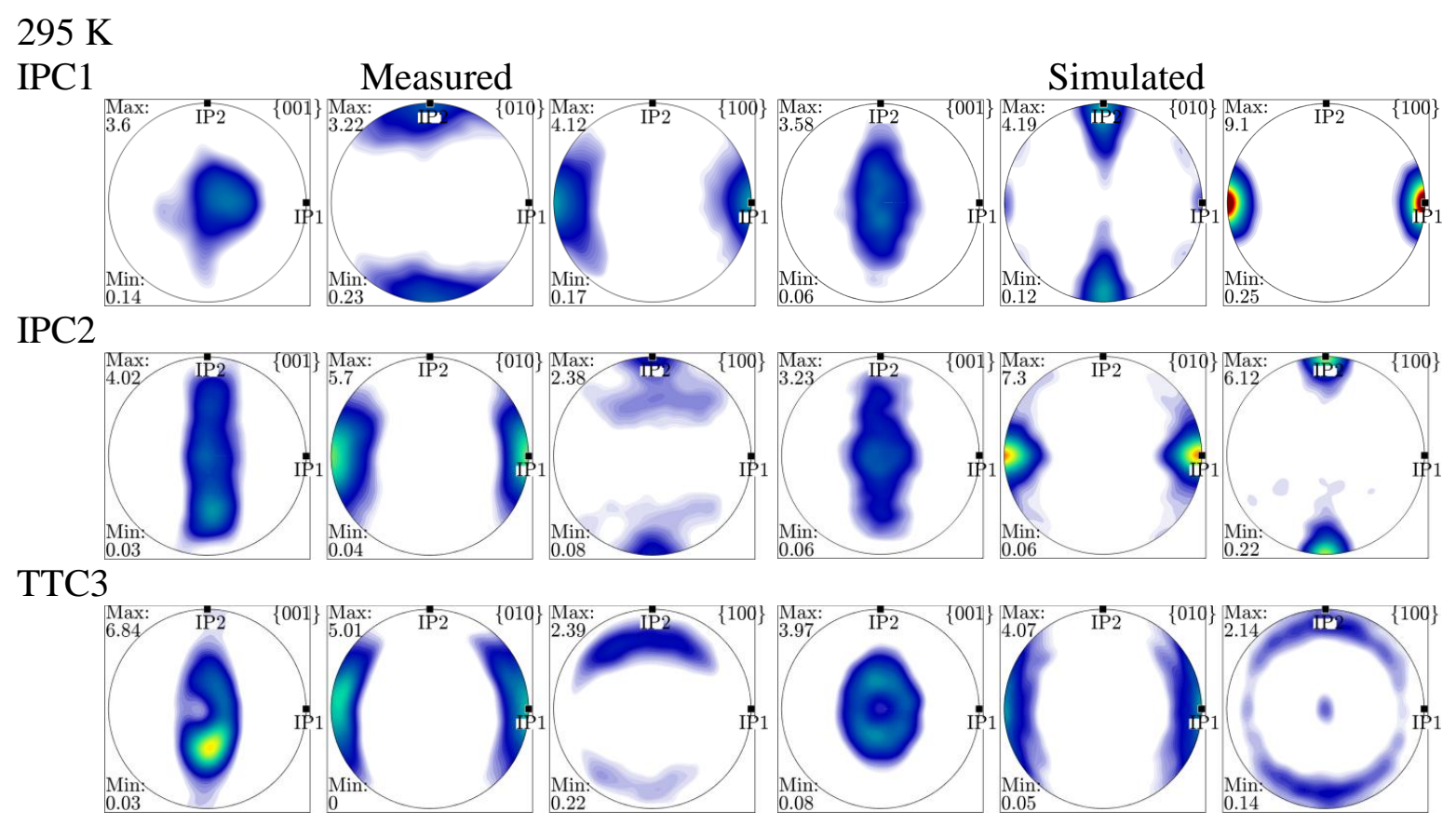




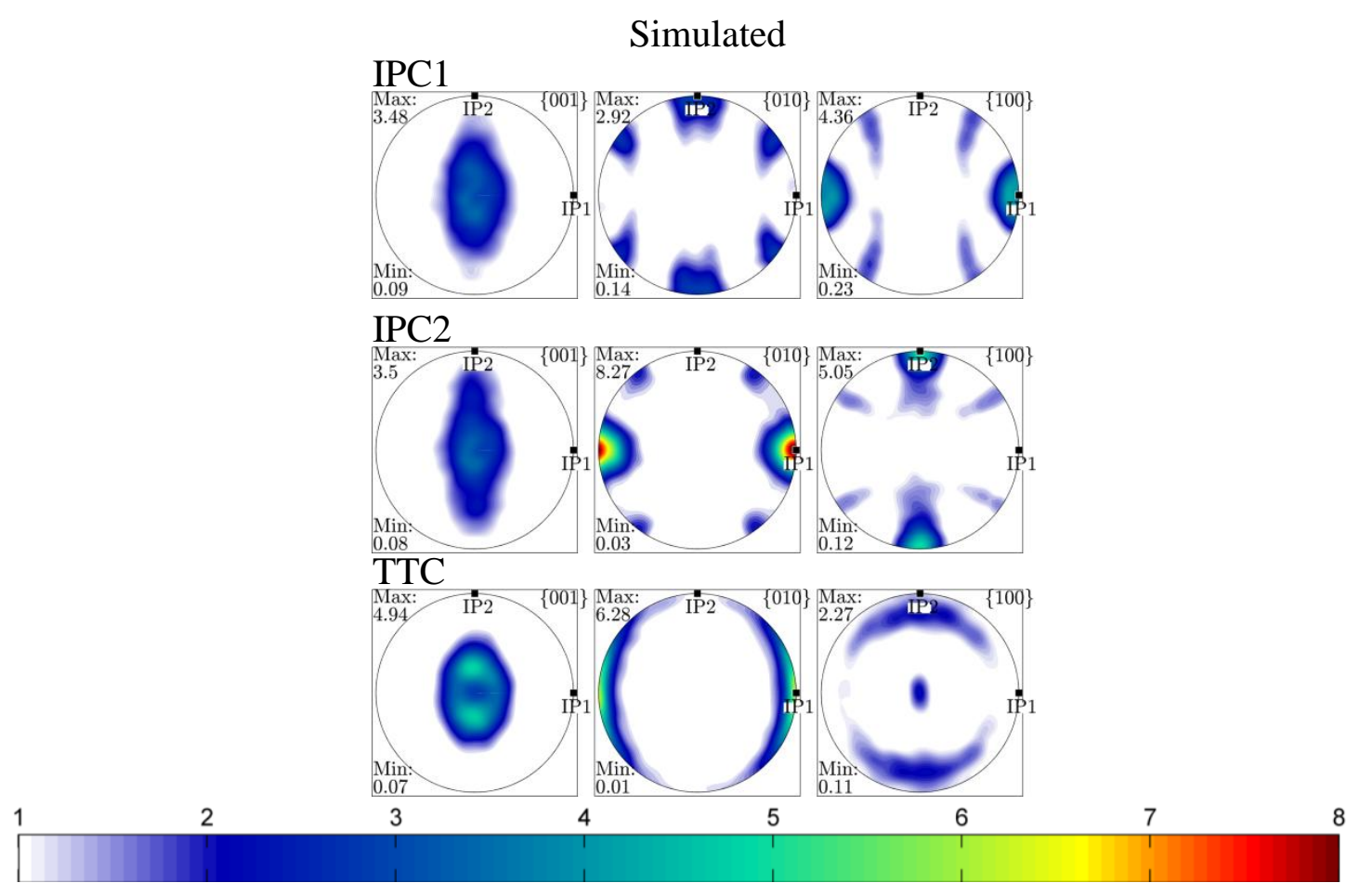

Fig. 12. Comparison of measured and predicted texture evolution during simple compression along the directions indicated in the figure of $\alpha-\mathrm{U}$ at room temperature and $573 \mathrm{~K}$.
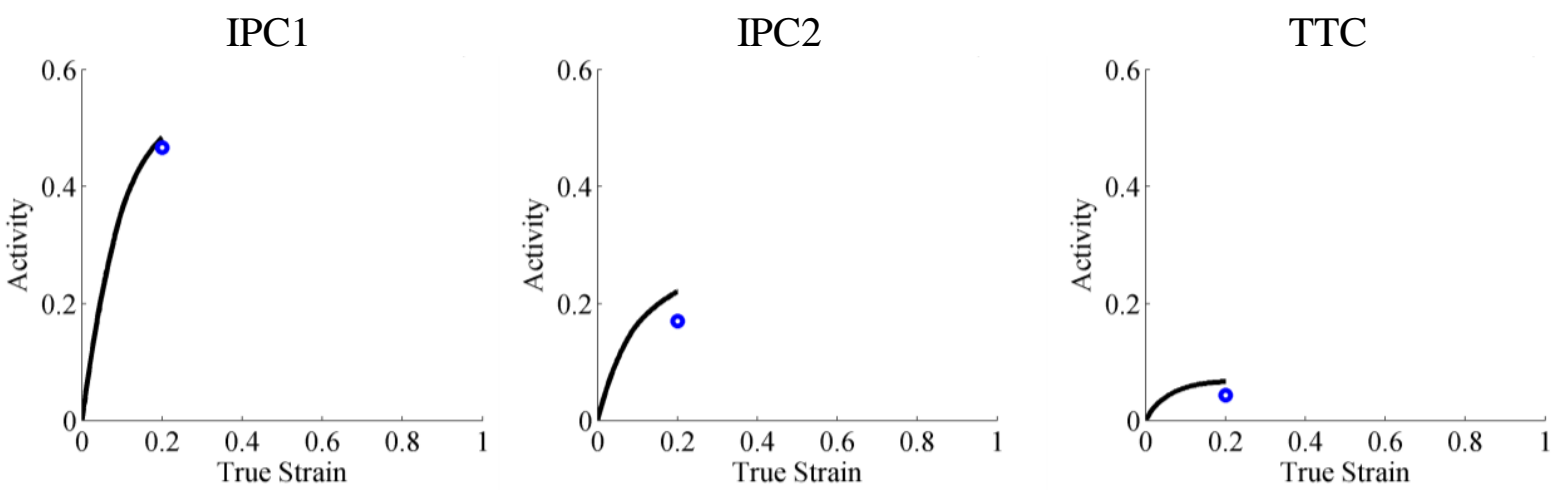

Fig. 13. Comparison of the twin volume fraction evolution between the model and experiment during simple compression at room temperature.

With the change of temperature, relative mode activities change and, consequently, corresponding texture features change. For comparison, we calculated the texture evolution at $573 \mathrm{~K}$, the same temperature that is used in rolling uranium. To first order, it appears that the texture evolution at room temperature is qualitatively similar to that at $573 \mathrm{~K}$. More noticeable differences lie in the $\{010\}$ pole figure and a few extra components that appear in the $\{100\}$ pole 
figure. This would suggest that the predominant slip and twinning modes did not change appreciably with the increase in temperature; however, because the number of slip and twinning modes is large, it is not necessarily the case.

The relative activities of each mode involved in the texture development can be estimated using the model. In Fig. 14, we present the calculated relative activities of the different slip modes as a function of straining in simple compression for all three loading directions. In TTC, we observe that roof slip decreases in activity as temperature increases from room temperature to $573 \mathrm{~K}$, while wall and floor slip maintain approximately constant activity. Chimney $1 / 2\{110\}\langle 1 \overline{1} 0\rangle$ slip is enhanced as temperature increases. $\{130\}\langle 3 \overline{1} 0\rangle$ twinning is negligible. In the two in-plane compression directions, it is observed that twinning is reduced at the higher temperature and is replaced by $1 / 2\{110\}\langle 1 \overline{1} 0\rangle$ slip.

$295 \mathrm{~K}$
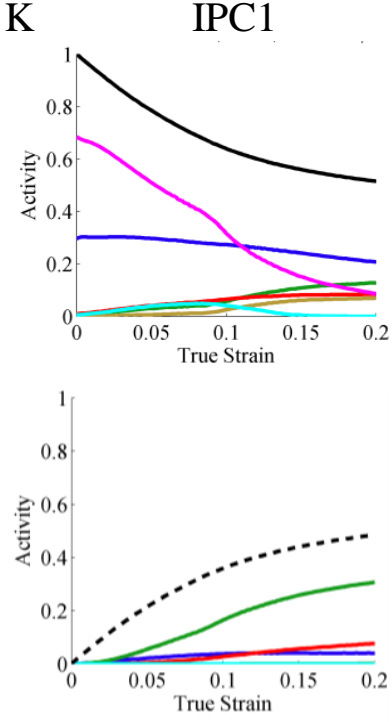

573K

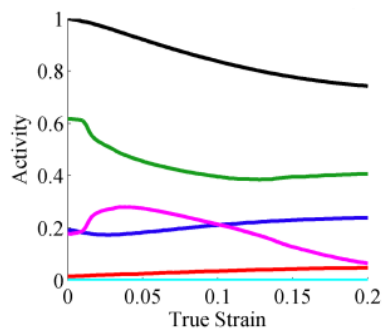

IPC2
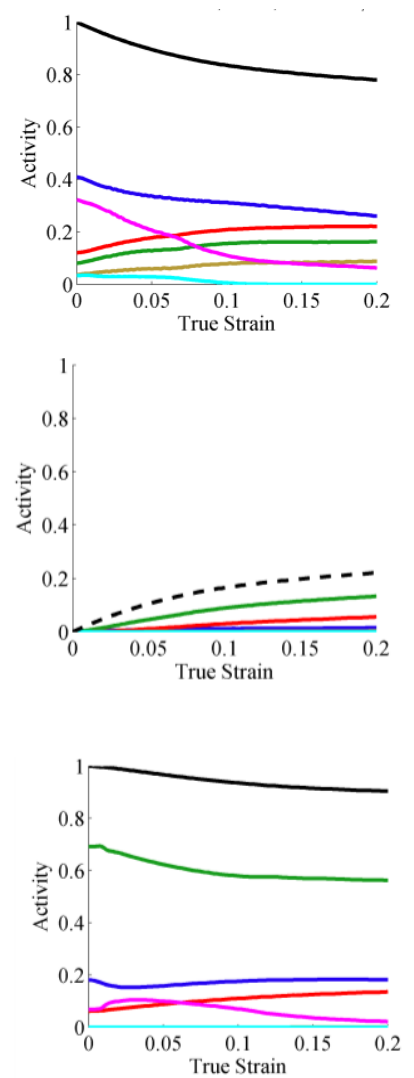

TTC
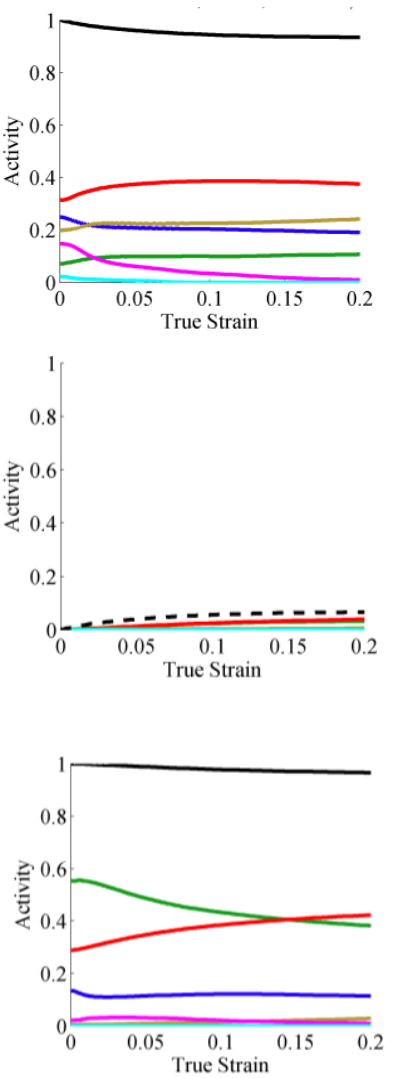

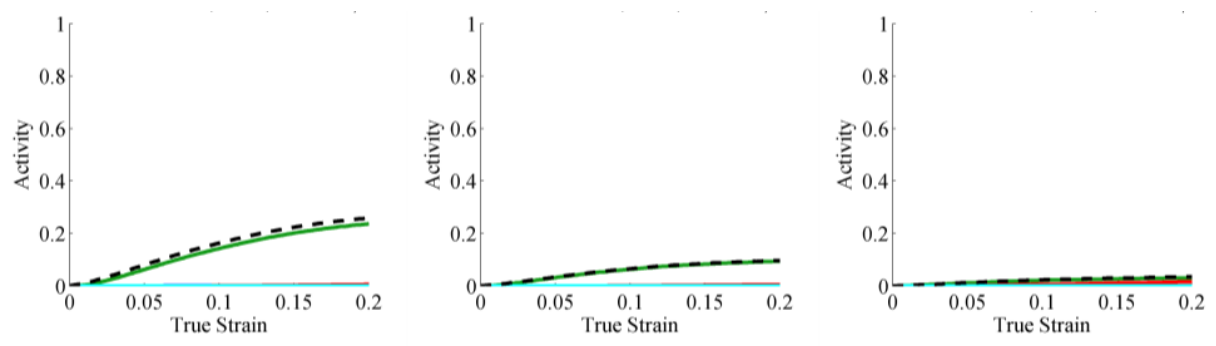

Fig. 14. Predicted relative activities of active deformation modes within parent grains (top rows) and within twins (bottom rows) during simple compression at room temperature and $573 \mathrm{~K}$. Also plotted are the corresponding parent material (top row) and twinned material (bottom row) volume fraction. The legend is the same as in Fig. 6.

\subsubsection{Lattice reorientations due to twinning}

As before in plane strain compression, we used the VPSC model to determine which grains are prone to $\{130\}\langle 3 \overline{1} 0\rangle$ deformation twinning in simple compression. Figures $15 \mathrm{a}$ and $\mathrm{b}$ present the maps of twinnable grains (blue) on pole figures for room temperature simple compression in IPC2 and IPC1 after a strain of 0.2. Generally it is found that the twinnable grains have their $\{010\}$ poles oriented along the compression direction. Twinning reorients the lattice such that $\{010\}$ poles lie normal to the compression direction or the $\{100\}$ poles lie along it.

(a)

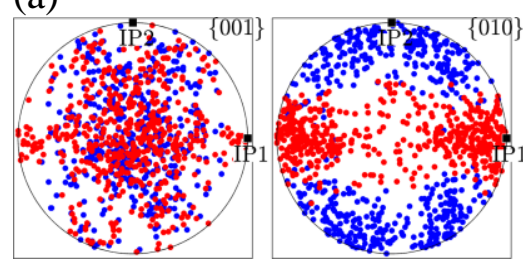

(b)

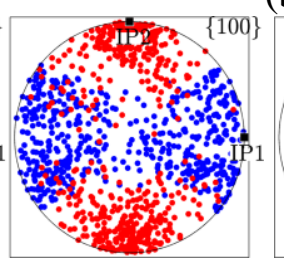

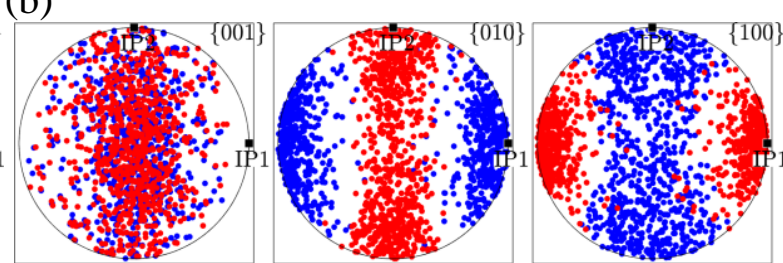

Fig. 15. Parent grains that develop twins (blue) and their twins (red) after simple compression along (a) IPC2 and (b) IPC1 for the initially textured material in Fig. 1a after a compression strain of 0.2. The poles after simple compression in TTC are not shown since twinning is not active during TTC.

\subsubsection{Lattice reorientation tendencies}

The textures studied in the prior sections are consequences of the activation of different combinations of slip and twinning. We see each mode provides a different amount to accommodating strain. However, from such an analysis, how much each mode contributes to lattice reorientation is not obvious. As done for plane strain compression, we use the VPSC model and Sachs-like model to relate observed crystal reorientations seen in texture evolution with the activated slip modes. Together these models are used to calculate the reorientation 
tendencies (averaged over all grains) observed for a given slip mode and twinning mode during simple compression along the TT, IPC1 and IPC2. Figure 16 presents the lattice reorientations for simple compression in TTC, IPC2, and IPC1.

For TTC (Fig. 16a), floor slip causes $\{001\}$ poles originally away from the TT direction to rotate towards the TT direction and form a ring about the TT compression direction. In addition, roof slip has an opposing effect on $\{001\}$ pole reorientation to floor slip, and since both are active in TTC, we see that these peaks become diffuse. The two peaks on $\{001\}$ pole figure are a consequence of the initial texture. Although chimney slip is active at $573 \mathrm{~K}$, it does not result in a reorientation in the $\{001\}$ pole figure. Thus, with an increase in temperature, the increase in intensities of two peaks on $\{001\}$ pole figure is mainly caused by lower activity of roof slip. In TTC in the $\{010\}$ pole figure, we can see a reorientation towards the center is caused by roof slip. Floor slip does not cause a reorientation in the (010) pole figure. In the (001) pole figure, we can see the increase of the peak at the center, which is an outcome of the increased activity of chimney slip. From this mapping, we can better understand the changes in texture development as temperature increases. For instance, increased activity of chimney and decreased activity of roof slip at higher temperatures cause increase of radius of ring formed on the $\{010\}$ pole figure and stronger peaks in the IP1 direction.

For the IPC2 test (Fig. 16b), in the $\{001\}$ pole figure we see the formation of peaks towards the IP2 direction. These are caused by floor slip. In the $\{010\}$ pole figure, $\{130\}\langle 3 \overline{1} 0\rangle$ twinning and chimney slip result in the formation of the distinct peaks in the IP1 direction. It should be noted that chimney slip is predominantly active within twins, along with some floor slip. The analysis suggests that roof slip would have the opposite effect and cause weakening of these peaks. In the $\{100\}$ pole figure, the formation of the distinct peaks in the IP1 direction is again caused by $\{130\}\langle 3 \overline{1} 0\rangle$ twinning and chimney slip. Any observed weakening of these poles and reorientation towards the center would be caused by floor slip. This analysis can explain why the textures do not change much as temperature increases from room temperature to $573 \mathrm{~K}$. The change in temperature and texture causes a reduction in the activity of $\{130\}\langle 3 \overline{1} 0\rangle$ twinning and roof slip, while chimney slip activity increases substantially. Therefore the distinct poles in the $\{010\}$ and $\{100\}$ pole figures at high temperature are solely caused by the large activity of chimney slip and the absence of roof slip, which has the opposite effect of chimney slip. 
In the IPC1 test (Fig. 16c), in the $\{001\}$ pole figure, we do not observe an evolution from the initial $\{001\}$ pole figure. This outcome occurs because neither twinning nor wall and chimney slip cause reorientations on this pole figure. In the $\{010\}$ pole figure, we find that the formation of peaks along the IP2 direction is caused by $\{130\}\langle 3 \overline{1} 0\rangle$ twinning and that the large activity of chimney slip within the twins results in further reorientation towards IP2. Wall slip reorients the grains so that they are favorably oriented for $\{130\}\langle 3 \overline{1} 0\rangle$ twinning and in a sense helps increase twinning activity. In the $\{100\}$ pole figure, again we find that the formation of the peaks along the IP1 direction is caused by the large twin volume fraction and large activity of chimney slip within twins.

In closing, it is worth mentioning that the added benefit of the reorientation analysis presented here could be to refine the hardening parameters in such a way to promote different slip and twinning systems over others, which would in turn result in the simulated textures to be closer to the measured textures.

(a) TTC

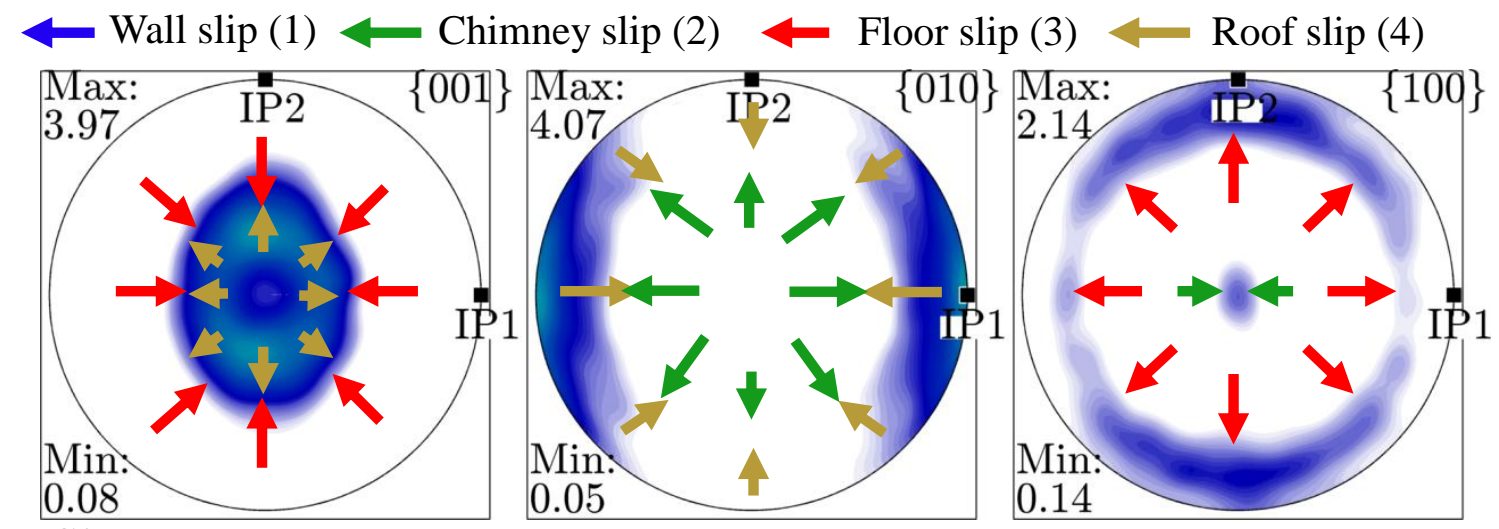

(b) IPC2

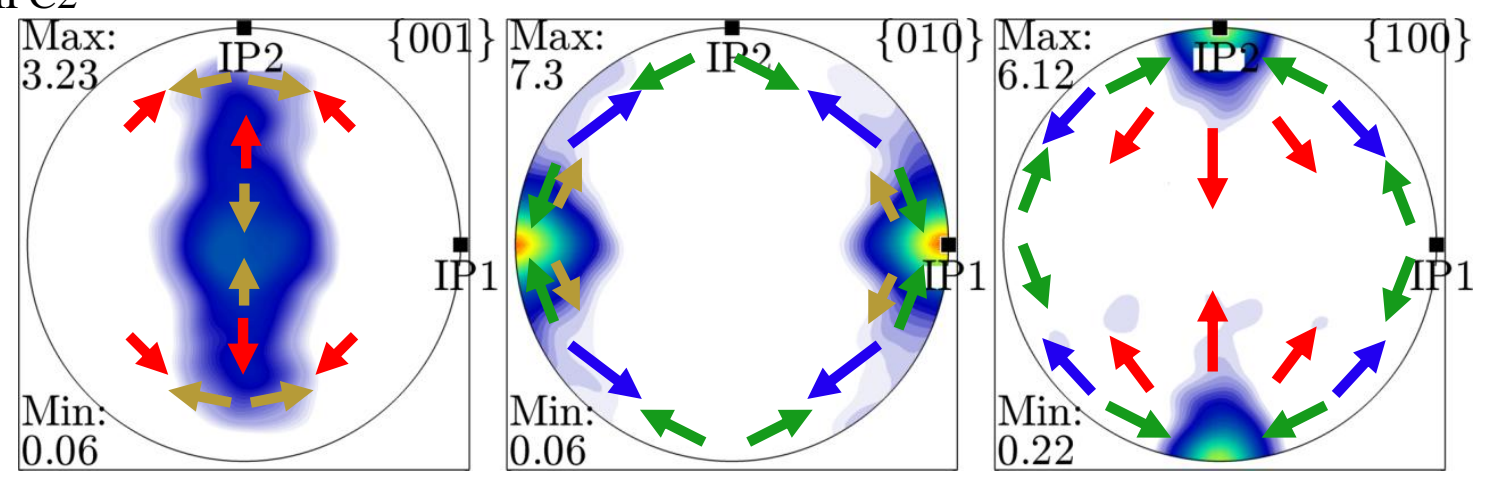


(c) IPC1
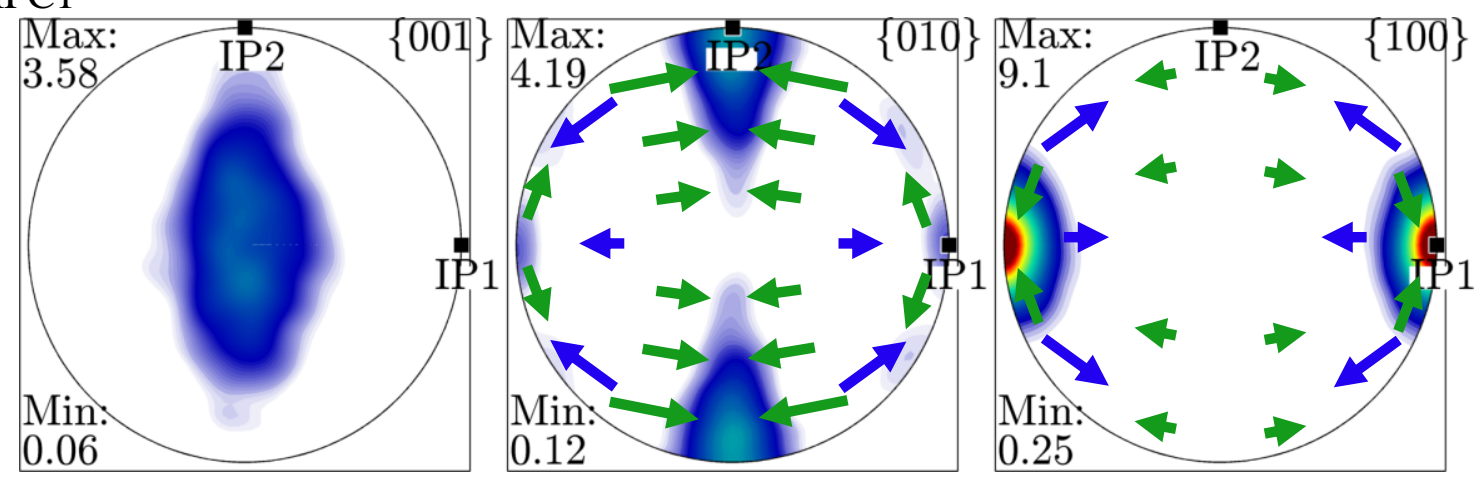

Fig. 16. Summary of lattice reorientations due to slip in simple compression as indicated in the figure. Note that twinning reorients the lattice such that $\{010\}$ poles lie normal to the compression direction and the $\{100\}$ poles lie along it.

\section{Conclusions}

In this work, we use polycrystal plasticity to understand the slip and twinning modes responsible for the development of particular texture components in PSC and simple compression of $\alpha-\mathrm{U}$. To this end, we study lattice reorientation produced by experimentally calibrated and validated models. In PSC, the analysis finds that floor slip (001)[100] causes the formation of two pronounced (001) texture peaks $10-15^{\circ}$ away from the normal direction toward the rolling direction. This result for $\alpha-\mathrm{U}$, (with a c/a ratio of 1.734), has interesting parallels to

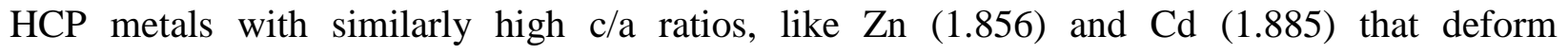
predominantly by basal slip. These HCP metals tend to also exhibit textures with (0001) poles tilted $\pm 15-25^{\circ}$ away from the normal toward the rolling direction. The analysis reveals that substantially different reorientation trends are present in simple compression from those in PSC at a given temperature. While twinning is largely suppressed for TTC at $573 \mathrm{~K}$, it is profuse in PSC at the same temperature. In-plane room temperature deformation is dominated by twinning, which decreases with increasing temperature. Although texture formation in simple compression does not exhibit large sensitivity to temperature, the relative activity of active slip modes changes with temperature. We rationalize that this is possible in $\alpha-\mathrm{U}$ because the large number of active deformation modes contribute to texture evolution.

\section{Acknowledgements}

M. Z. acknowledges support from the CEPS Graduate Fellowships program at the University of New Hampshire (UNH). M. K. acknowledges subcontract, NO. 277871, granted by Los Alamos 
National Laboratory to UNH. I. J. B. and R. J. M. acknowledge support by a Laboratory Directed Research and Development grant 20140630ER.

\section{References}

[1] M.H. Yoo, Slip modes of alpha uranium, Journal of Nuclear Materials, 26 (1968) 307-318.

[2] R.W. Cahn, Twinning and slip in $\alpha$-uranium, Acta Crystallographica, 4 (1951) 470.

[3] J.S. Daniel, B. Lesage, P. Lacombe, The influence of temperature on slip and twinning in uranium, Acta Metallurgica, 19 (1971) 163-173.

[4] M. Knezevic, R.J. McCabe, C.N. Tomé, R.A. Lebensohn, S.R. Chen, C.M. Cady, G.T. Gray Iii, B. Mihaila, Modeling mechanical response and texture evolution of $\alpha$-uranium as a function of strain rate and temperature using polycrystal plasticity, International Journal of Plasticity, 43 (2013) 70-84.

[5] M. Knezevic, L. Capolungo, C.N. Tomé, R.A. Lebensohn, D.J. Alexander, B. Mihaila, R.J. McCabe, Anisotropic stress-strain response and microstructure evolution of textured $\alpha$-uranium, Acta Materialia, 60 (2012) 702-715.

[6] C.A. Calhoun, E. Garlea, R.P. Mulay, T.A. Sisneros, S.R. Agnew, Investigation of the effect of thermal residual stresses on deformation of $\alpha$-uranium through neutron diffraction measurements and crystal plasticity modeling, Acta Materialia, 85 (2015) 168-179.

[7] M. Knezevic, J. Crapps, I.J. Beyerlein, D.R. Coughlin, K.D. Clarke, R.J. McCabe, Anisotropic modeling of structural components using embedded crystal plasticity constructive laws within finite elements, International Journal of Mechanical Sciences, 105 (2016) 227-238. [8] M. Knezevic, R.J. McCabe, R.A. Lebensohn, C.N. Tomé, B. Mihaila, Finite element implementation of a self-consistent polycrystal plasticity model: Application to $\alpha$-uranium, Proceedings: Volume 2: Materials Properties, Characterization, and Modeling TMS (The Minerals, Metals \& Materials Society), in, 2012, pp. 789-796.

[9] M. Knezevic, R.J. McCabe, R.A. Lebensohn, C.N. Tomé, C. Liu, M.L. Lovato, B. Mihaila, Integration of self-consistent polycrystal plasticity with dislocation density based hardening laws within an implicit finite element framework: Application to low-symmetry metals, Journal of the Mechanics and Physics of Solids, 61 (2013) 2034-2046.

[10] M. Knezevic, T. Nizolek, M. Ardeljan, I.J. Beyerlein, N.A. Mara, T.M. Pollock, Texture evolution in two-phase $\mathrm{Zr} / \mathrm{Nb}$ lamellar composites during accumulative roll bonding, International Journal of Plasticity, 57 (2014) 16-28.

[11] M. Ardeljan, I.J. Beyerlein, M. Knezevic, A dislocation density based crystal plasticity finite element model: Application to a two-phase polycrystalline HCP/BCC composites, Journal of the Mechanics and Physics of Solids, 66 (2014) 16-31.

[12] J.S. Carpenter, T. Nizolek, R.J. McCabe, M. Knezevic, S.J. Zheng, B.P. Eftink, J.E. Scott, S.C. Vogel, T.M. Pollock, N.A. Mara, I.J. Beyerlein, Bulk texture evolution of nanolamellar Zr$\mathrm{Nb}$ composites processed via accumulative roll bonding, Acta Materialia, 92 (2015) 97-108.

[13] M. Zecevic, M. Knezevic, I.J. Beyerlein, C.N. Tomé, An elasto-plastic self-consistent model with hardening based on dislocation density, twinning and de-twinning: Application to strain path changes in HCP metals, Materials Science and Engineering: A, 638 (2015) 262-274.

[14] A. Chapuis, Q. Liu, Simulations of texture evolution for HCP metals: Influence of the main slip systems, Computational Materials Science, 97 (2015) 121-126. 
[15] K. Linga Murty, I. Charit, Texture development and anisotropic deformation of zircaloys, Progress in Nuclear Energy, 48 (2006) 325-359.

[16] M.J. Philippe, Texture Formation in Hexagonal Materials Materials Science Forum, 157 162 (1994) 1337-1350.

[17] M. Knezevic, I.J. Beyerlein, T. Nizolek, N.A. Mara, T.M. Pollock, Anomalous Basal Slip Activity in Zirconium under High-strain Deformation, Materials Research Letters, 1 (2013) 133140.

[18] M. Knezevic, A. Levinson, R. Harris, R.K. Mishra, R.D. Doherty, S.R. Kalidindi, Deformation twinning in AZ31: Influence on strain hardening and texture evolution, Acta Materialia, 58 (2010) 6230-6242.

[19] M. Knezevic, N.W. Landry, Procedures for reducing large datasets of crystal orientations using generalized spherical harmonics, Mechanics of Materials, 88 (2015) 73-86.

[20] R.J. McCabe, L. Capolungo, P.E. Marshall, C.M. Cady, C.N. Tomé, Deformation of wrought uranium: Experiments and modeling, Acta Materialia, 58 (2010) 5447-5459. [21] S.I. Wright, M.M. Nowell, S.P. Lindeman, P.P. Camus, M. De Graef, M.A. Jackson, Introduction and comparison of new EBSD post-processing methodologies, Ultramicroscopy, 159, Part 1 (2015) 81-94.

[22] M. Knezevic, M. Zecevic, I.J. Beyerlein, J.F. Bingert, R.J. McCabe, Strain rate and temperature effects on the selection of primary and secondary slip and twinning systems in $\mathrm{HCP}$ Zr, Acta Materialia, 88 (2015) 55-73.

[23] R.A. Lebensohn, C.N. Tomé, A self-consistent anisotropic approach for the simulation of plastic deformation and texture development of polycrystals: Application to zirconium alloys, Acta Metallurgica et Materialia, 41 (1993) 2611-2624.

[24] M. Knezevic, M. Jahedi, Y.P. Korkolis, I.J. Beyerlein, Material-based design of the extrusion of bimetallic tubes, Computational Materials Science, 95 (2014) 63-73.

[25] M. Knezevic, R.A. Lebensohn, O. Cazacu, B. Revil-Baudard, G. Proust, S.C. Vogel, M.E. Nixon, Modeling bending of $\alpha$-titanium with embedded polycrystal plasticity in implicit finite elements, Materials Science and Engineering: A, 564 (2013) 116-126.

[26] J.D. Eshelby, The determination of the elastic field of an ellipsoidal inclusion, and related problems, Proc R. Soc. Lond. A, 241 (1957) 376-396.

[27] C. Tomé, C. Necker, R. Lebensohn, Mechanical anisotropy and grain interaction in recrystallized aluminum, Metallurgical and Materials Transactions A, 33 (2002) 2635-2648. [28] I.J. Beyerlein, J.S. Carpenter, A. Hunter, L.S. Tóth, W. Skrotzki, Nano-enabled orientation alignment via extreme shear strains, Scripta Materialia, 98 (2015) 52-55.

[29] J.W. Hutchinson, Bounds and self-consistent estimates for creep of polycrystalline materials, Proceedings of the Royal Society of London. Series A, Mathematical and Physical Sciences, (1976) 101-126.

[30] R.J. Asaro, A. Needleman, Texture development and strain hardening in rate dependent polycrystals, Acta Metallurgica et Materialia, 33 (1985) 923-953.

[31] M. Knezevic, B. Drach, M. Ardeljan, I.J. Beyerlein, Three dimensional predictions of grain scale plasticity and grain boundaries using crystal plasticity finite element models, Computer Methods in Applied Mechanics and Engineering, 277 (2014) 239-259.

[32] S. Kok, A.J. Beaudoin, D.A. Tortorelli, A polycrystal plasticity model based on the mechanical threshold, International Journal of Plasticity, 18 (2002) 715-741.

[33] G. Proust, C.N. Tomé, G.C. Kaschner, Modeling texture, twinning and hardening evolution during deformation of hexagonal materials, Acta Materialia, 55 (2007) 2137-2148. 
[34] M. Ardeljan, M. Knezevic, T. Nizolek, I.J. Beyerlein, N.A. Mara, T.M. Pollock, A study of microstructure-driven strain localizations in two-phase polycrystalline HCP/BCC composites using a multi-scale model, International Journal of Plasticity, 74 (2015) 35-57.

[35] I.J. Beyerlein, C.N. Tomé, A dislocation-based constitutive law for pure Zr including temperature effects, International Journal of Plasticity, 24 (2008) 867-895.

[36] M. Jahedi, M. Ardeljan, I.J. Beyerlein, M.H. Paydar, M. Knezevic, Enhancement of orientation gradients during simple shear deformation by application of simple compression, Journal of Applied Physics, 117 (2015) 214309.

[37] M. Knezevic, I.J. Beyerlein, D.W. Brown, T.A. Sisneros, C.N. Tomé, A polycrystal plasticity model for predicting mechanical response and texture evolution during strain-path changes: Application to beryllium, International Journal of Plasticity, 49 (2013) 185-198. [38] M. Knezevic, I.J. Beyerlein, M.L. Lovato, C.N. Tomé, A.W. Richards, R.J. McCabe, A strain-rate and temperature dependent constitutive model for BCC metals incorporating nonSchmid effects: Application to tantalum-tungsten alloys, International Journal of Plasticity, 62 (2014) 93-104.

[39] M. Knezevic, J.S. Carpenter, M.L. Lovato, R.J. McCabe, Deformation behavior of the cobalt-based superalloy Haynes 25: Experimental characterization and crystal plasticity modeling, Acta Materialia, 63 (2014) 162-168.

[40] H. Mecking, U.F. Kocks, Kinetics of flow and strain-hardening., Acta Metallurgica et Materialia, 29 (1981) 1865-1875.

[41] R. Madec, B. Devincre, L.P. Kubin, From Dislocation Junctions to Forest Hardening, Physical Review Letters, 89 (2002) 255508.

[42] U. Essmann, H. Mughrabi, Annihilation of dislocations during tensile and cyclic deformation and limits of dislocation densities, Philosophical Magazine A, 40 (1979) 731-756. [43] M. Zecevic, M. Knezevic, A dislocation density based elasto-plastic self-consistent model for the prediction of cyclic deformation: Application to Al6022-T4, International Journal of Plasticity, 72 (2015) 200-217.

[44] R.W. Cahn, Plastic deformation of alpha-uranium; twinning and slip, Acta Metallurgica, 1 (1953) 49-70.

[45] R.G. Anderson, J.W. Bishop, The effect of neutron irradiation and thermal cycling on permanent deformations in uranium under load, in: Symposium on uranium and graphite, The Institute of Metals, London 1962, pp. 17-23.

[46] E.S. Fisher, H.J. McSkimin, Adiabatic elastic moduli of single crystal alpha Uranium, Journal of Applied Physics, 29 (1958) 1473 - 1484.

[47] R.A. Lebensohn, C.N. Tomé, A self-consistent viscoplastic model: prediction of rolling textures of anisotropic polycrystals, Materials Science and Engineering: A, 175 (1994) 71-82. [48] A.D. Rollett, Comparison of experimental and theoretical texture development in alphauranium, in: T.C. Lowe, A.D. Rollett, P.S. Follansbee, G.S. Daehn (Eds.) Symposium on Modeling the Deformation of Crystalline Solids, TMS, Warrendale, PA, 1991, pp. 361-368. [49] D.W. Brown, M.A.M. Bourke, B. Clausen, D.R. Korzekwa, R.C. Korzekwa, R.J. McCabe, T.A. Sisneros, D.F. Teter, Temperature and direction dependence of internal strain and texture evolution during deformation of uranium, Materials Science and Engineering: A, 512 (2009) 6775.

[50] C.S. Choi, M. Staker, Neutron diffraction texture study of deformed uranium plates, J Mater Sci, 31 (1996) 3397-3402. 
[51] M. Ardeljan, R.J. McCabe, I.J. Beyerlein, M. Knezevic, Explicit incorporation of deformation twins into crystal plasticity finite element models, Computer Methods in Applied Mechanics and Engineering, 295 (2015) 396-413.

[52] M. Zecevic, M. Knezevic, I.J. Beyerlein, R.J. McCabe, Texture formation in orthorhombic alpha-uranium under simple compression and rolling to high strains, Journal of Nuclear Materials, 473 (2016) 143-156.

[53] M. Zecevic, R.J. McCabe, M. Knezevic, A new implementation of the spectral crystal plasticity framework in implicit finite elements, Mechanics of Materials, 84 (2015) 114-126.

[54] M. Zecevic, R.J. McCabe, M. Knezevic, Spectral database solutions to elasto-viscoplasticity within finite elements: Application to a cobalt-based FCC superalloy, International Journal of Plasticity, 70 (2015) 151-165.

[55] U.F. Kocks, C.N. Tomé, H.-R. Wenk, Texture and Anisotropy, Cambridge University Press, Cambridge, 1998.

[56] Y. Wang, J. Huang, Texture analysis in hexagonal materials, Materials Chemistry and Physics, 81 (2003) 11-26.

[57] A. Sheikh-Ali, D. Molodov, H. Garmestani, Magnetically induced texture development in zinc alloy sheet, Scripta Materialia, 46 (2002) 857-862.

[58] M.J. Philippe, F. Wagner, F.E. Mellab, C. Esling, J. Wegria, Modelling of texture evolution for materials of hexagonal symmetry-I. Application to zinc alloys, Acta Metallurgica et Materialia, 42 (1994) 239-250.

[59] F. Zhang, G. Vincent, Y.H. Sha, L. Zuo, J.J. Fundenberger, C. Esling, Experimental and simulation textures in an asymmetrically rolled zinc alloy sheet, Scripta Materialia, 50 (2004) 1011-1015.

[60] G.I. Taylor, Plastic strain in metals, Journal of the Institute of Metals, 62 (1938) 307-324. [61] M. Knezevic, S.R. Kalidindi, D. Fullwood, Computationally efficient database and spectral interpolation for fully plastic Taylor-type crystal plasticity calculations of face-centered cubic polycrystals, International Journal of Plasticity, 24 (2008) 1264-1276.

[62] M. Knezevic, S.R. Kalidindi, R.K. Mishra, Delineation of first-order closures for plastic properties requiring explicit consideration of strain hardening and crystallographic texture evolution, International Journal of Plasticity, 24 (2008) 327-342.

[63] M. Knezevic, H.F. Al-Harbi, S.R. Kalidindi, Crystal plasticity simulations using discrete Fourier transforms, Acta Materialia, 57 (2009) 1777-1784.

[64] M. Knezevic, D.J. Savage, A high-performance computational framework for fast crystal plasticity simulations, Computational Materials Science, 83 (2014) 101-106.

[65] D.J. Savage, M. Knezevic, Computer implementations of iterative and non-iterative crystal plasticity solvers on high performance graphics hardware, Computational Mechanics 56 (2015) 677-690.

[66] C.N. Tomé, R.A. Lebensohn, Manual for Code Viscoplastic Self-consistent (version 7c). (2011).

[67] T. Fast, M. Knezevic, S.R. Kalidindi, Application of microstructure sensitive design to structural components produced from hexagonal polycrystalline metals, Computational Materials Science, 43 (2008) 374-383.

[68] B.S. Fromm, B.L. Adams, S. Ahmadi, M. Knezevic, Grain size and orientation distributions: Application to yielding of $\alpha$-titanium, Acta Materialia, 57 (2009) 2339-2348. [69] J.B. Shaffer, M. Knezevic, S.R. Kalidindi, Building texture evolution networks for deformation processing of polycrystalline fcc metals using spectral approaches: Applications to 
process design for targeted performance, International Journal of Plasticity, 26 (2010) 11831194.

[70] H.F. Al-Harbi, M. Knezevic, S.R. Kalidindi, Spectral approaches for the fast computation of yield surfaces and first-order plastic property closures for polycrystalline materials with cubictriclinic textures, CMC: Computers, Materials, \& Continua, 15 (2010) 153-172.

[71] G. Sachs, Zur ableitung einer fliessbedingung, in: Mitteilungen der deutschen Materialprüfungsanstalten, Springer, 1929, pp. 94-97.

[72] M. Knezevic, S.R. Kalidindi, Fast computation of first-order elastic-plastic closures for polycrystalline cubic-orthorhombic microstructures, Computational Materials Science, 39 (2007) 643-648.

[73] L.R.A. Tomé C.N., Manual for Code Viscoplastic Self-consistent (version 7c). (2011). 